
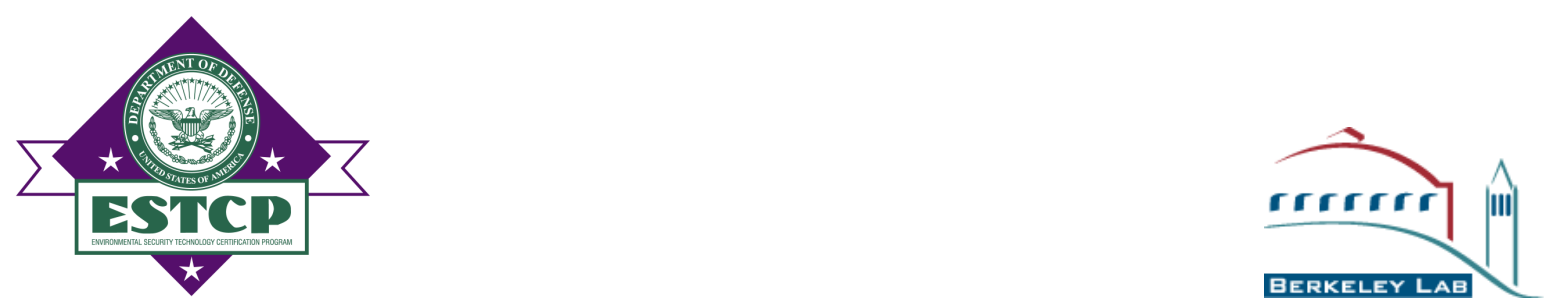

DEMONSTRATION REPORT

\title{
A MULTISENSOR SYSTEM FOR THE DETECTION AND \\ CHARACTERIZATION OF UXO
}

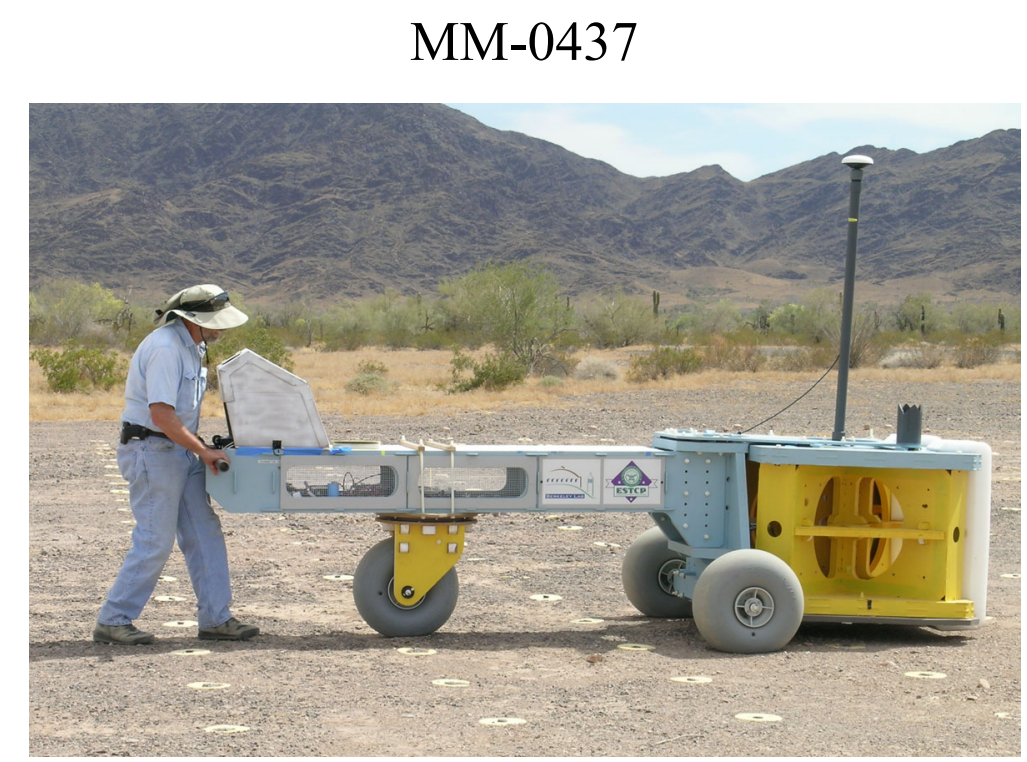

SITE LOCATION:

U.S. ARMY YUMA PROVING GROUND

DEMONSTRATOR:

LAWRENCE BERKELEY NATIONAL LABORATORY

ONE CYCLOTRON ROAD, MS: 90R1116

BERKELEY, CA 94720

p.o.c. Erika Gasperikova, egasperikova@lbl.gov, 510-486-4930

TECHNOLOGY TYPE/PLATFORM:

BUD/CART

JUNE 2006 


\section{BUD DESCRIPTION}

The Berkeley UXO discriminator (BUD) (Figure 1) is a portable Active Electromagnetic (AEM) system for UXO detection and characterization that quickly determines the location, size, and symmetry properties of a suspected UXO. The BUD comprises of three orthogonal transmitters that "illuminate" a target with fields in three independent directions in order to stimulate the three polarization modes that, in general, characterize the target EM response. In addition, the $B U D$ uses eight pairs of differenced receivers for response recording. Eight receiver coils are placed horizontally along the two diagonals of the upper and lower planes of the two horizontal transmitter loops. These receiver coil pairs are located on symmetry lines through the center of the system and each pair sees identical fields during the on-time of the pulse in all of the transmitter coils. They are wired in opposition to produce zero output during the on-time of the pulses in three orthogonal transmitters. Moreover, this configuration dramatically reduces noise in the measurements by canceling the background electromagnetic fields (these fields are uniform over the scale of the receiver array and are consequently nulled by the differencing operation), and by canceling the noise contributed by the tilt of the receivers in the Earth's magnetic field, and greatly enhances receivers sensitivity to the gradients of the target response. The $B U D$ performs target characterization from a single position of the sensor platform above a target. 


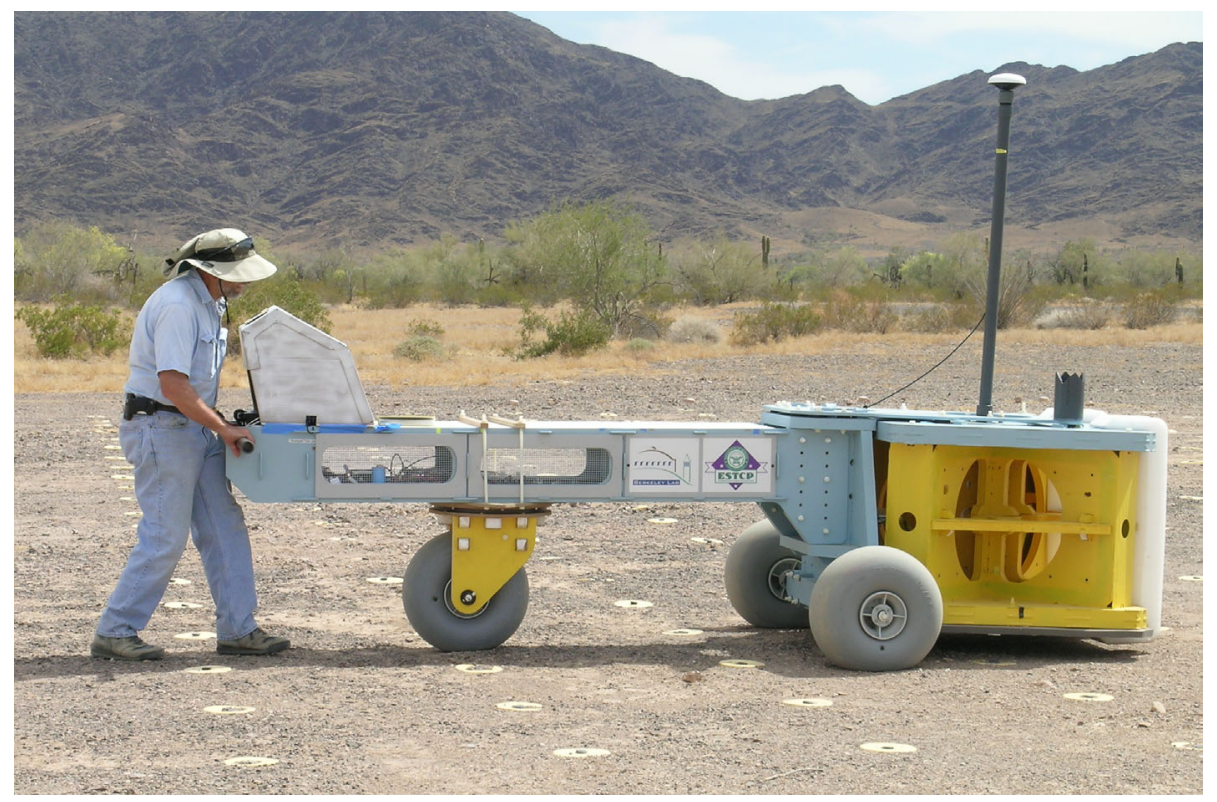

Figure 1. Berkeley UXO Discriminator (BUD)

$B U D$ was designed to detect and characterize UXO in the $20 \mathrm{~mm}$ to $155 \mathrm{~mm}$ size range for depths between 0 and $1 \mathrm{~m}$. The relationship between the object size and the depth at which it can be detected is illustrated in Figure 2. This curve was calculated for $B U D$ assuming that the receiver plane is $20 \mathrm{~cm}$ above the ground. Figure 2 shows that, for example, $B U D$ can detect and characterize an object with $10 \mathrm{~cm}$ diameter down to the depth of $90 \mathrm{~cm}$ with depth uncertainty of $10 \%$. Any objects buried at the depth more than $1 \mathrm{~m}$ have a low probability of detection. With existing algorithms in the system computer it is not possible to recover the principal polarizabilities of large objects close to the system. Detection of large shallow objects is assured, but at present real time discrimination for shallow objects is not. Post processing of the field data is required for shape discrimination of large shallow targets. Next generation of $B U D$ software will not have this limitation. Successful application of the inversion algorithm that solves for the target parameters is contingent upon resolution of this limitation. At the moment, interpretation software is developed for a single object only. In case of multiple objects the 
software indicates the presence of a cluster of objects but is unable to provide characteristics of each individual object.

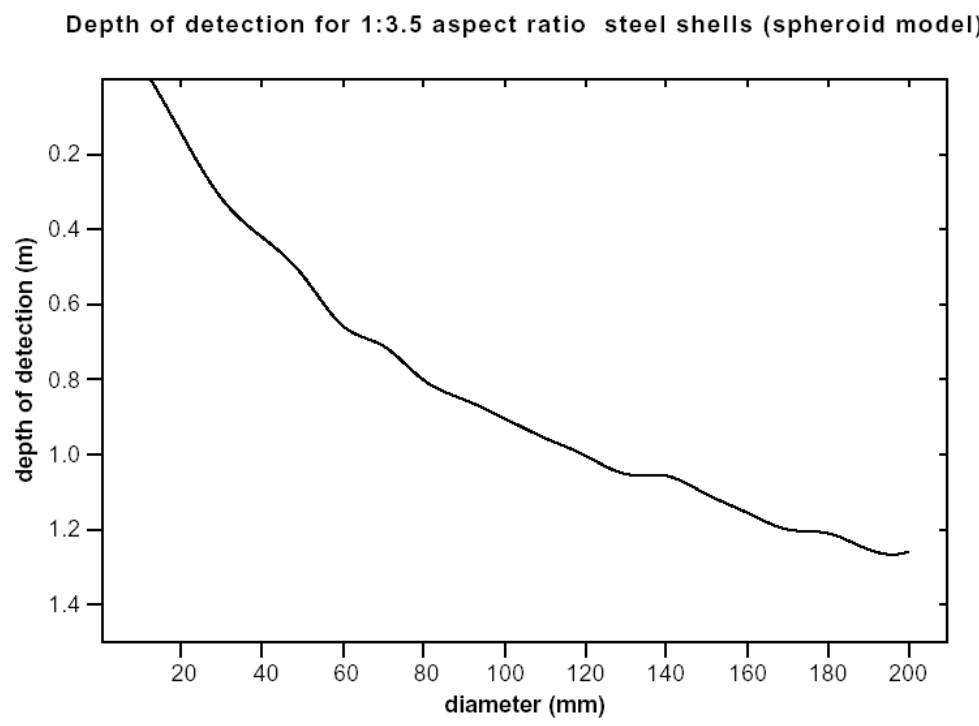

Figure 2. $10 \%$ uncertainty in location as a function of object diameter and depth of the detection for BUD with receivers $20 \mathrm{~cm}$ above the ground

\section{FIELD SURVEY - YUMA PROVING GROUND}

The objective of the Yuma Proving Ground (YPG) Demonstration was to acquire multicomponent data with $B U D$ over the Calibration Grid and the Blind Test Grid. The Calibration Grid is $30 \mathrm{~m}$ by $40 \mathrm{~m}$ area and consists of seventeen lanes and contains $132 \mathrm{UXO}$ objects. The Blind Test Grid is a 1600 square meter area and contains 400 cells, each of which can be occupied by UXO, clutter, or both, or it can be empty. The field test was conducted from May 1, 2006 to May 6, 2006. The Calibration Grid was surveyed with $1 \mathrm{~m}$ spacing along the lanes A through $\mathrm{K}$, and with $0.5 \mathrm{~m}$ spacing along the lanes $\mathrm{L}$ and $\mathrm{M}$. The Blind Test Grid was surveyed 
with $0.5 \mathrm{~m}$ spacing along the lanes, and $1 \mathrm{~m}$ spacing between lanes ( $\sim 3200$ measurements).

Figure 3 shows the measurement coverage for both grids.

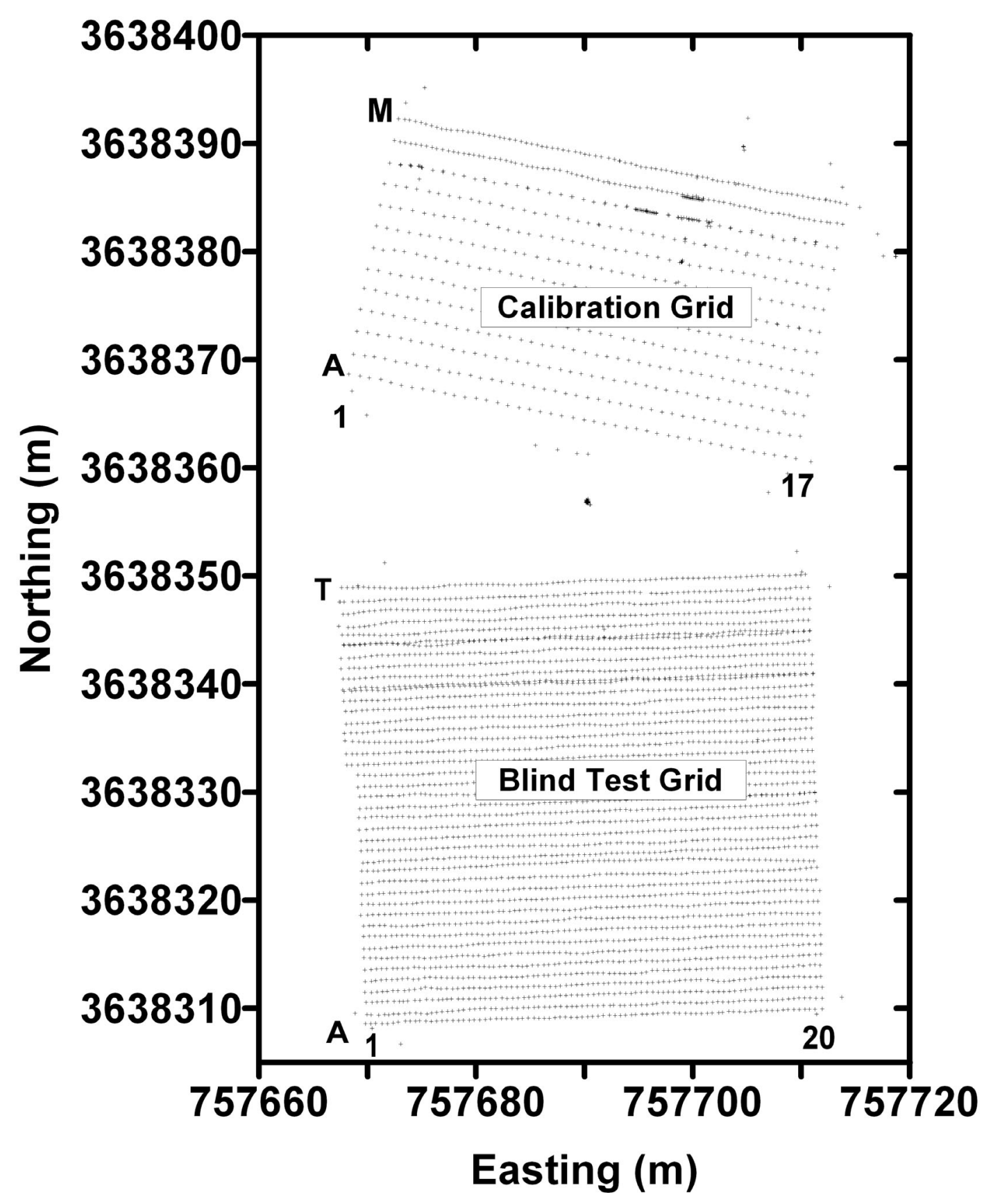

Figure 3: Measurement coverage over the Calibration and Blind Test Grids at Yuma Proving Ground. 


\section{DATA PROCESSING AND INTERPRETATION}

Raw field data are stored in the binary format. One file is created for one measurement point (station), which includes 8 channels of data for each of three transmitters, GPS and orientation information. Data are then stacked, normalized by a peak transmitter current, differenced with a reference trace (background transient) to remove system response transients, and averaged in logarithmically spaced bins with a half-sine weighting function. The resulting 24 channels of normalized responses are then inverted for candidate object position and principal polarizabilities as a function of time after transmitter shut-off. Data before $140 \mu$ s are ignored. For the data from the Blind Test Grid an object identification program matches measured equivalent dipole polarizabilities to a database of previous measurements of equivalent dipole polarizabilities of known objects and identifies a candidate object as the object(s) corresponding to the closest matching curves from the Calibration Grid. This is done by minimizing a robust loss function of the normalized absolute differences (residuals) between the measured values and those in the database weighted inversely by estimated uncertainty in polarizabilities. The database consists of all objects in the Calibration Grid and in the test pit.

Table 1 contains principal polarizabilities as a function of time of all objects from the Calibration Grid (132 entries). When the size-depth requirement (Figure 2) is satisfied, the polarizabilities are independent of the depth and orientation of the object. For large objects close to the system the principal polarizabilities curves vary depending on the orientation of the object. All objects, 
except A17 - a 12 pound shotput at the depth of $2.0 \mathrm{~m}$ were detected. ROC curve for the Calibration Grid is shown in Figure 4.

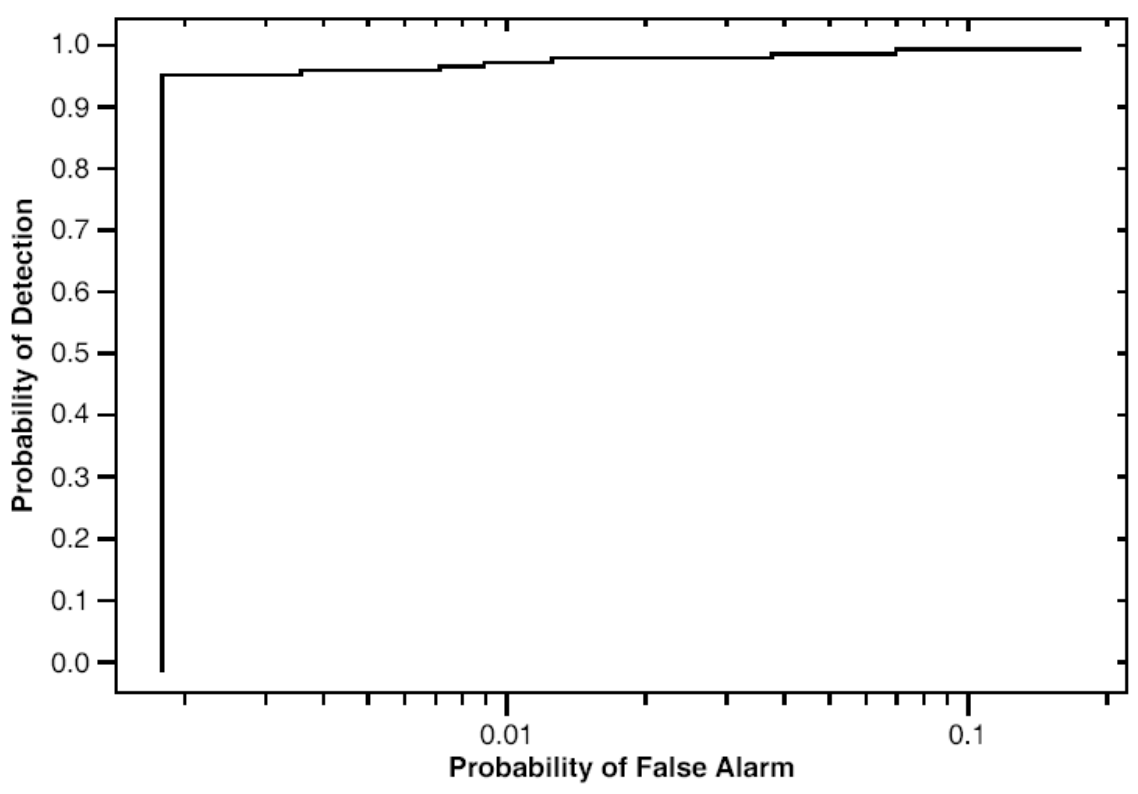

Figure 4: ROC curve for the Calibration Grid

Table 2 contains results from the Blind Test Grid. The table contains all the cells for which BUD indicates there is an object present, and a time weighted average signal strength is above $9 \times 10^{-12} \mathrm{Vs} / \mathrm{A}$ in response to the $\mathrm{Bz}$ transmitter or $4.5 \times 10^{-12} \mathrm{Vs} / \mathrm{A}$ in response to the either $\mathrm{Bx}$ or By transmitters, and the response level is not at a minimum compared to 1 meter forward or backwards along the survey line. For each cell that is not empty (228 occupied cells) the table contains a probability of the cell being occupied in column 1, and cell identification in column 2 . These probabilities are based on one minus the probability that the given response level would arise from random fluctuations of the background field in the absence of an object. From a total of 400 cells 228 cells or $57 \%$ are occupied. Figure 5 shows the probability of the cell being occupied in a graphical form. Cells with black plus symbols are empty. $85.1 \%$ from 228 cells 
have a probability higher than $90 \%$ that the cell is occupied (blue circles), $4.4 \%$ have probability between $75 \%$ and $90 \%$ (green squares), and $10.5 \%$ have probability between $30 \%$ and $75 \%$ (red diamonds). The results given in Table 2 and Figure 5 include objects listed for cells f_02n and f_18n, which are actually $1 \mathrm{~m}$ off the grid cell centers halfway between $\mathrm{f}_{-} 02$ and f_03, and between f_18 and f_19 respectively.

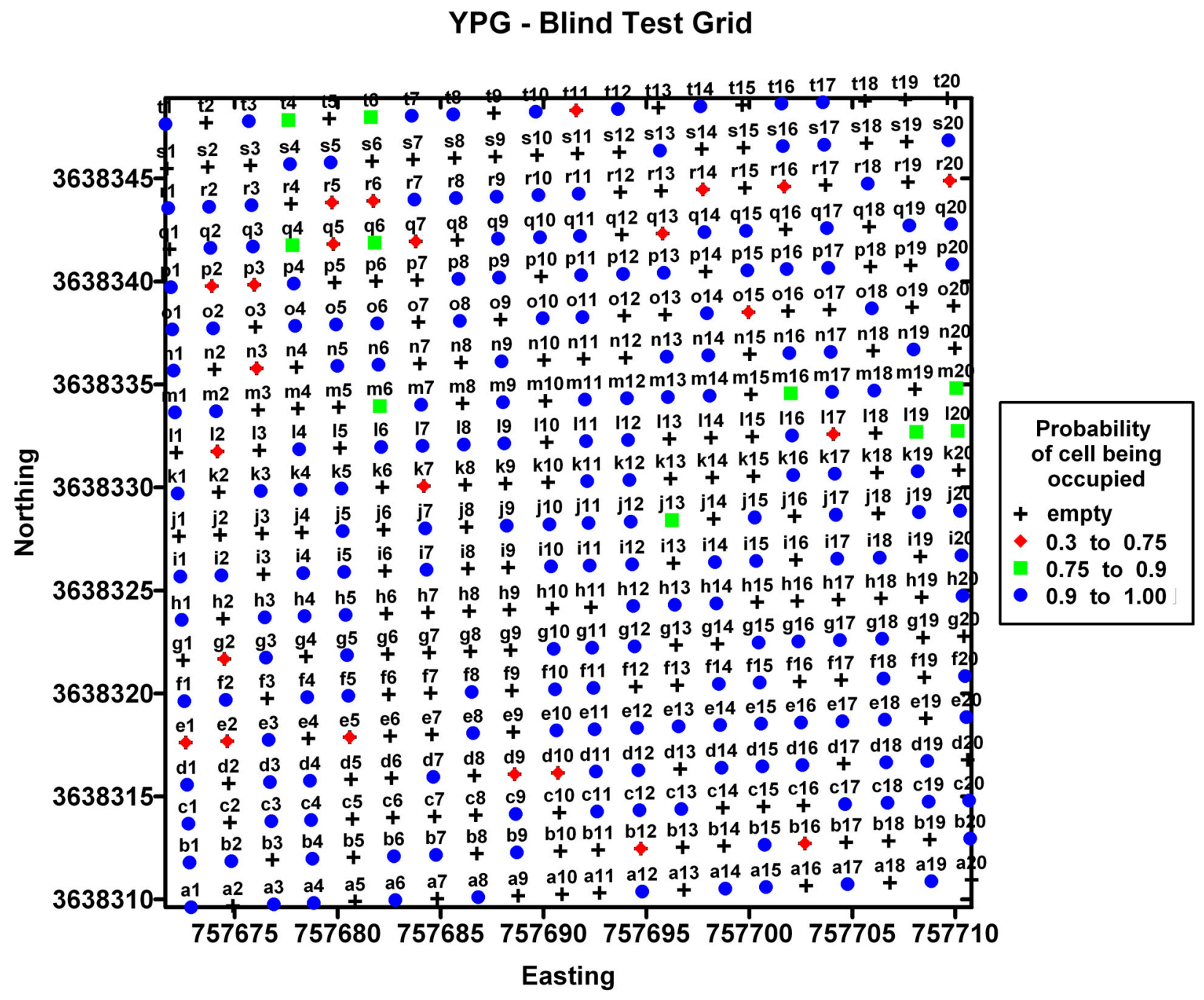

Figure 5: Blind Test Grid detection map 


\section{CONCLUSIONS}

The field survey with BUD at the Yuma Proving Ground was successful. BUD performed extremely well. BUD is easy to use and requires low maintenance - transmitter batteries last for three hours, while acquisition system batteries last for six hours. The system location was recorded with standard GPS with position accuracy about $9 \mathrm{~cm}$. We collected a large amount of multiple-transmitter multiple-receiver data over the Calibration Grid and Blind Test Grid. All data will be available to other researchers upon request. This report contains a table of polarizabilities curves for each object in the Calibration Grid, and detection results from the Blind Test Grid. We will submit another report with discrimination results from the Blind Test Grid after we address current limitations of our software described in Section 1. 
Table 1: Yuma Proving Ground - Calibration Grid

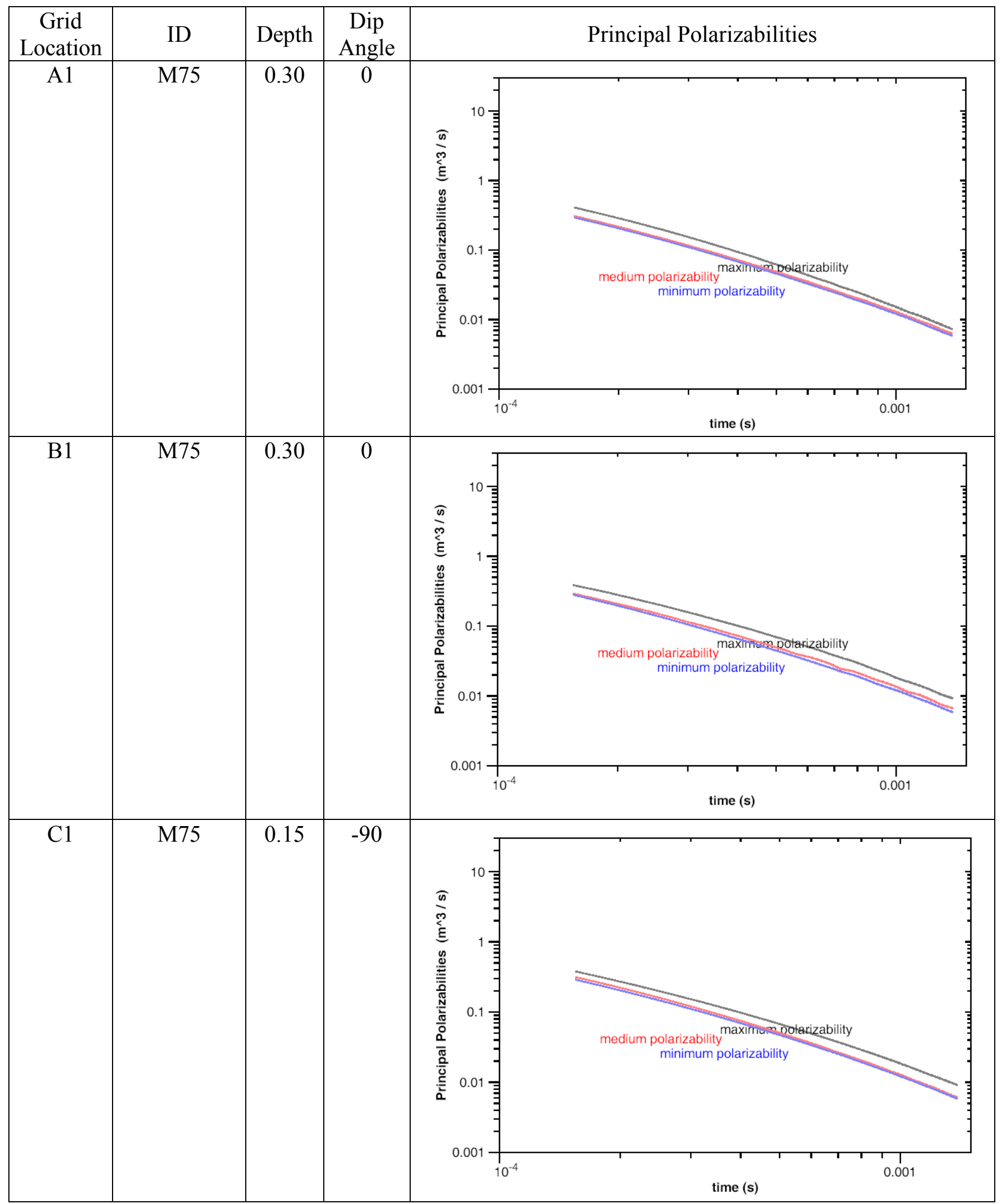




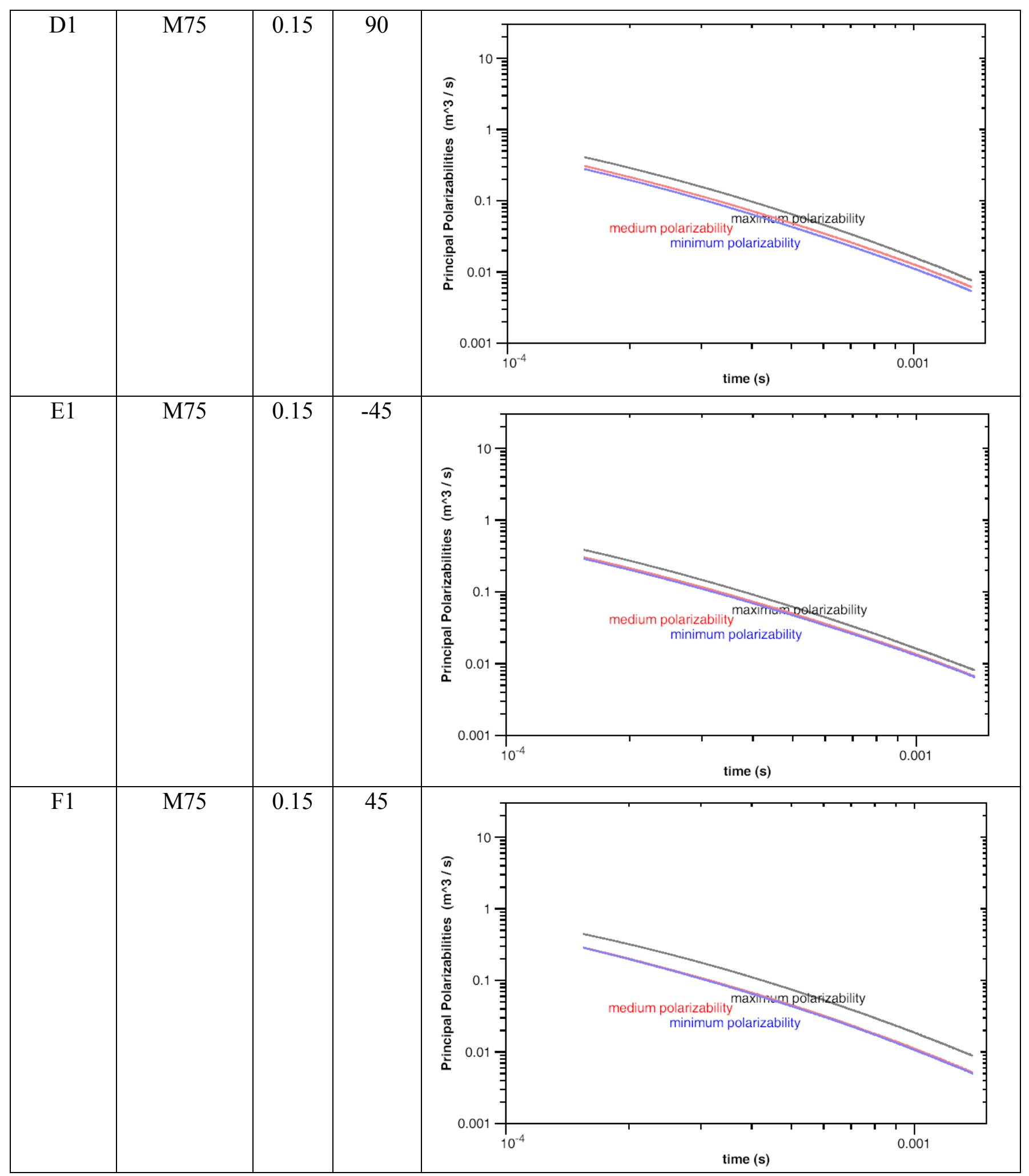




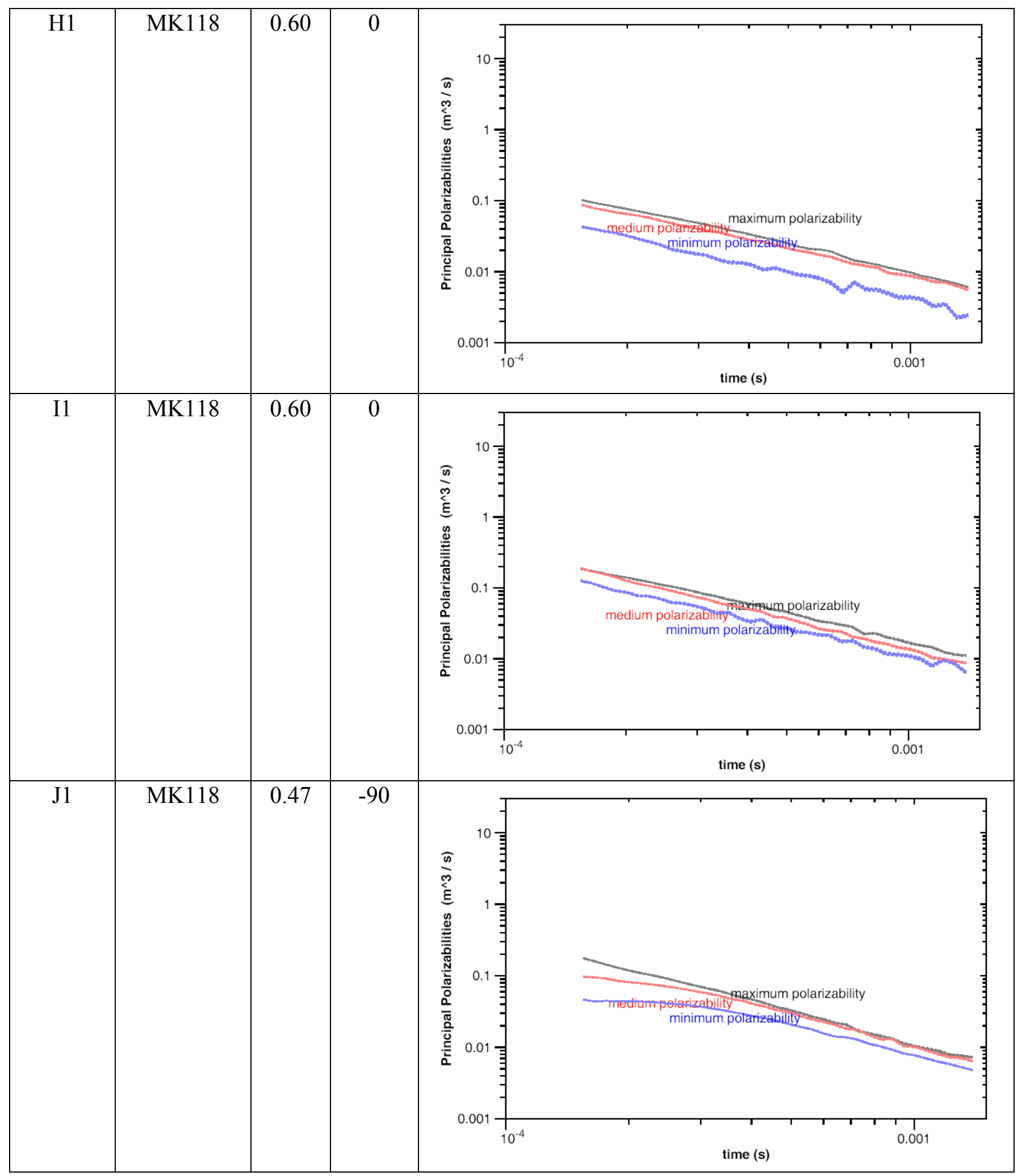




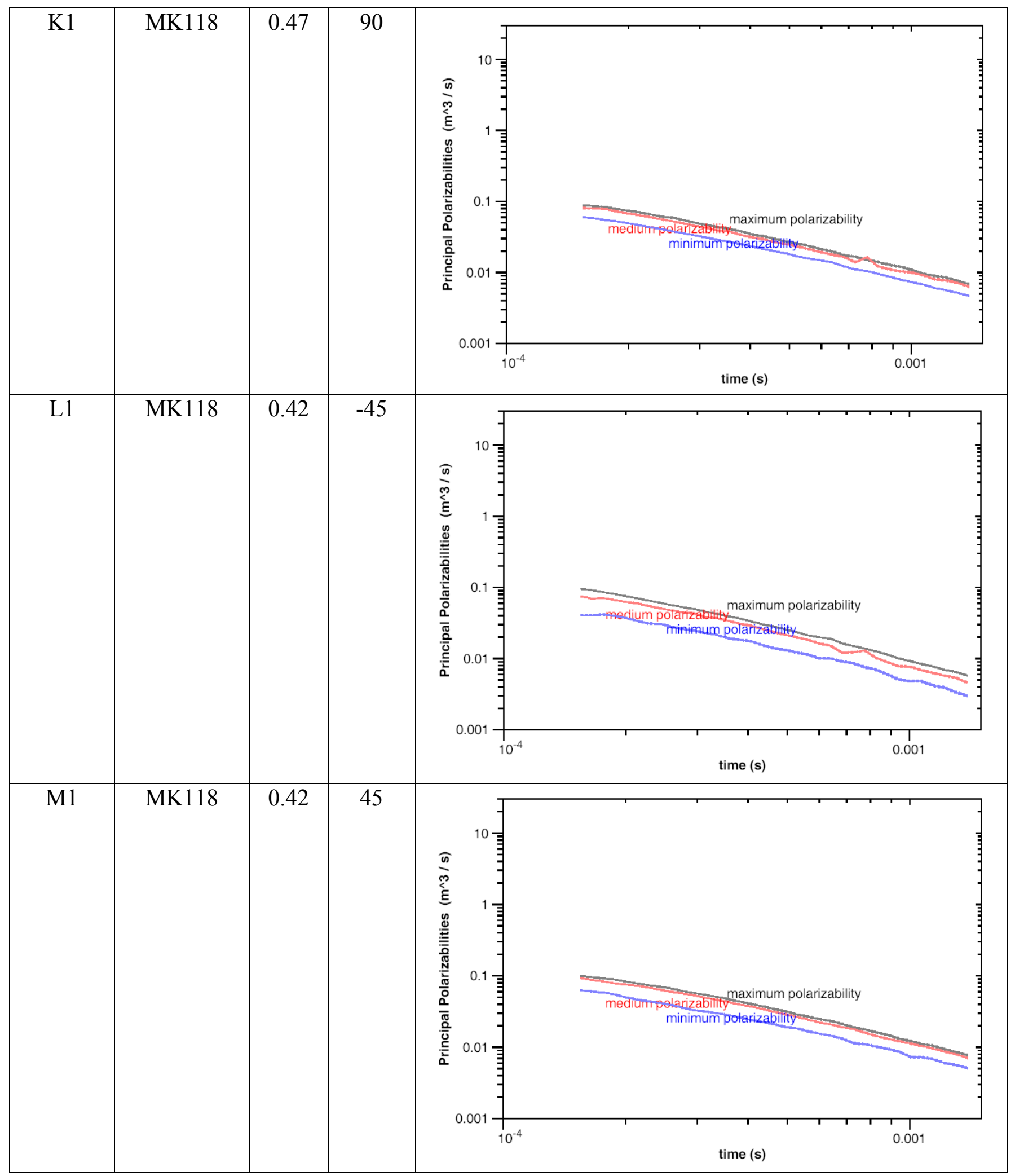




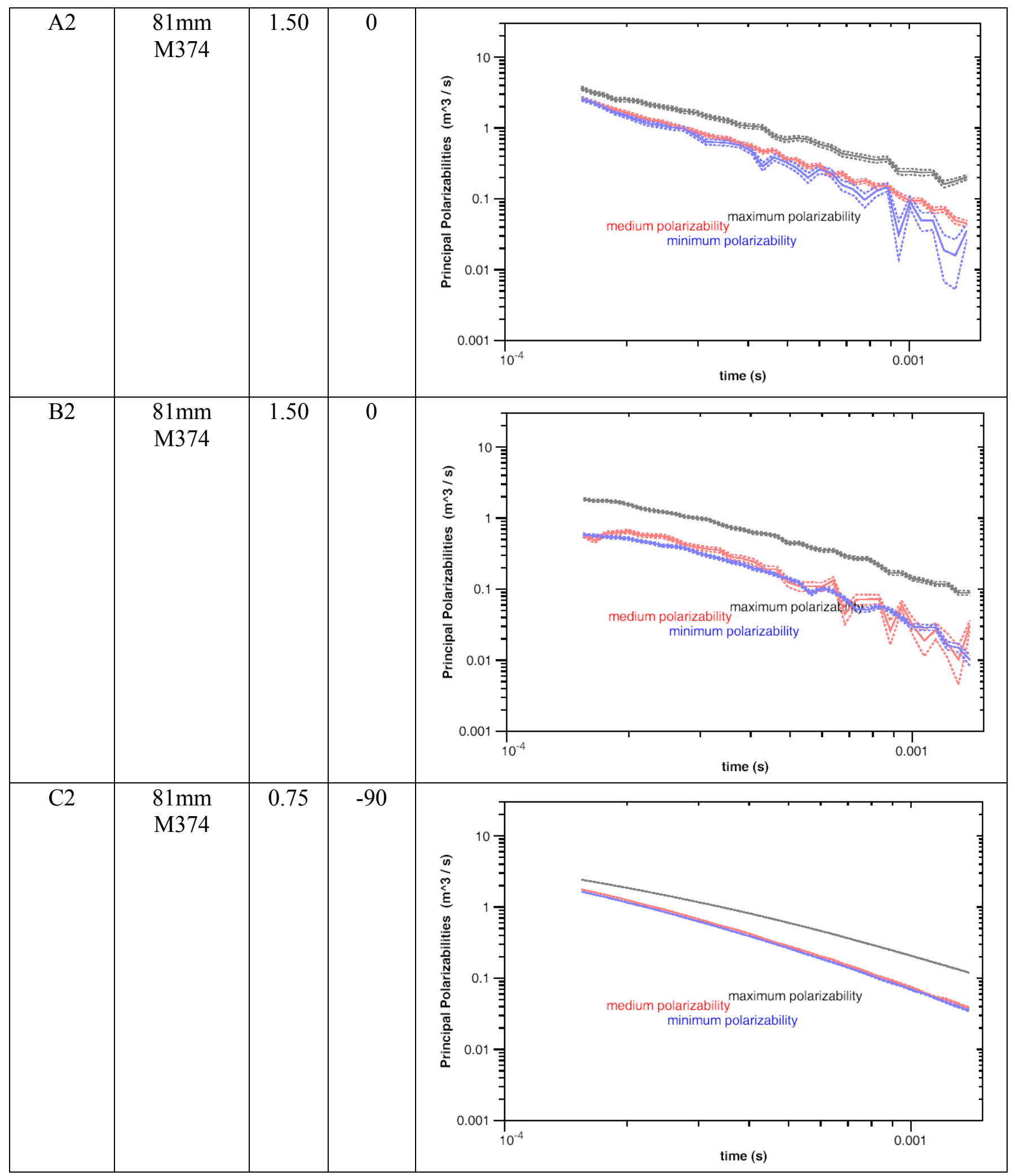




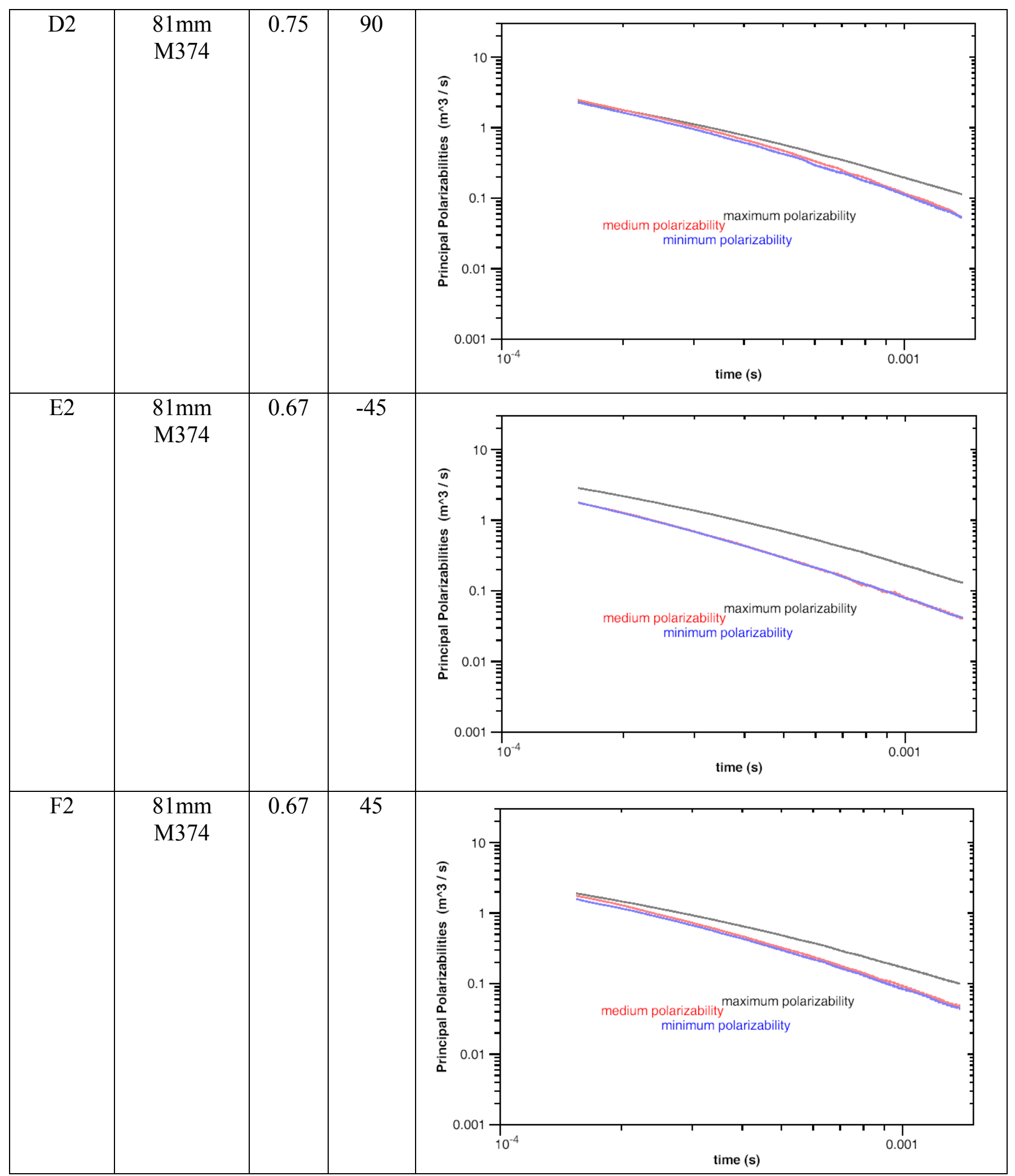




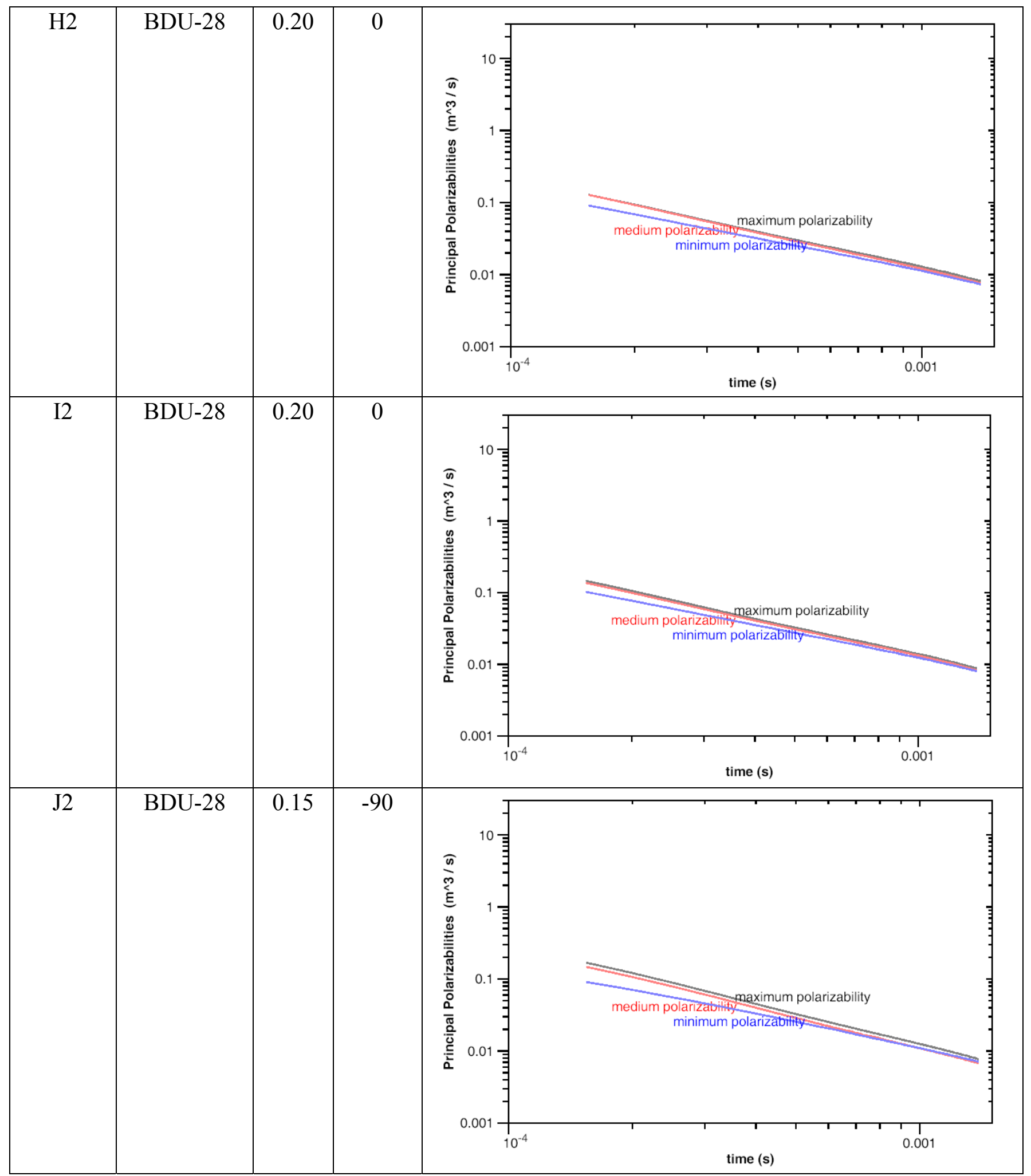




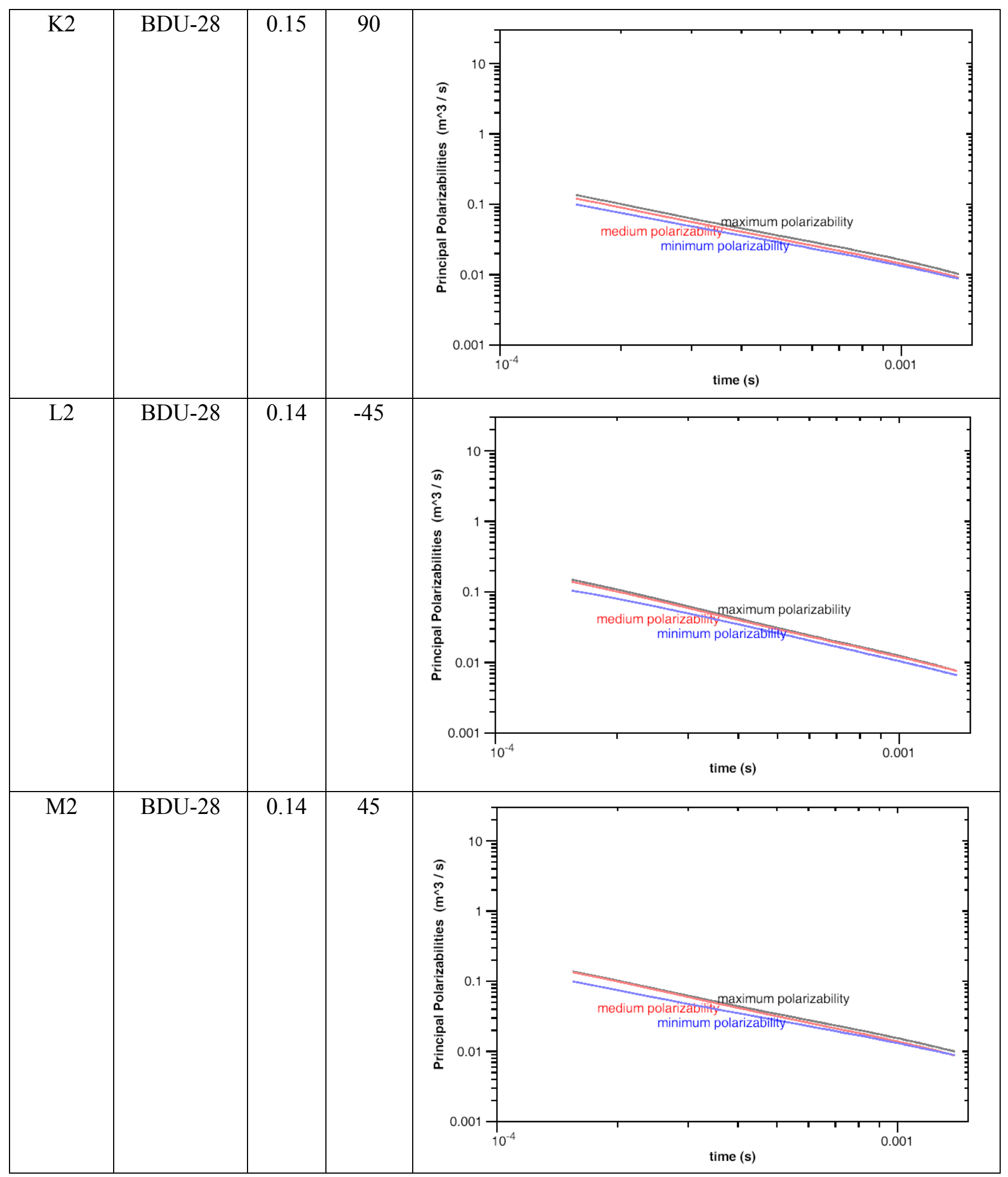




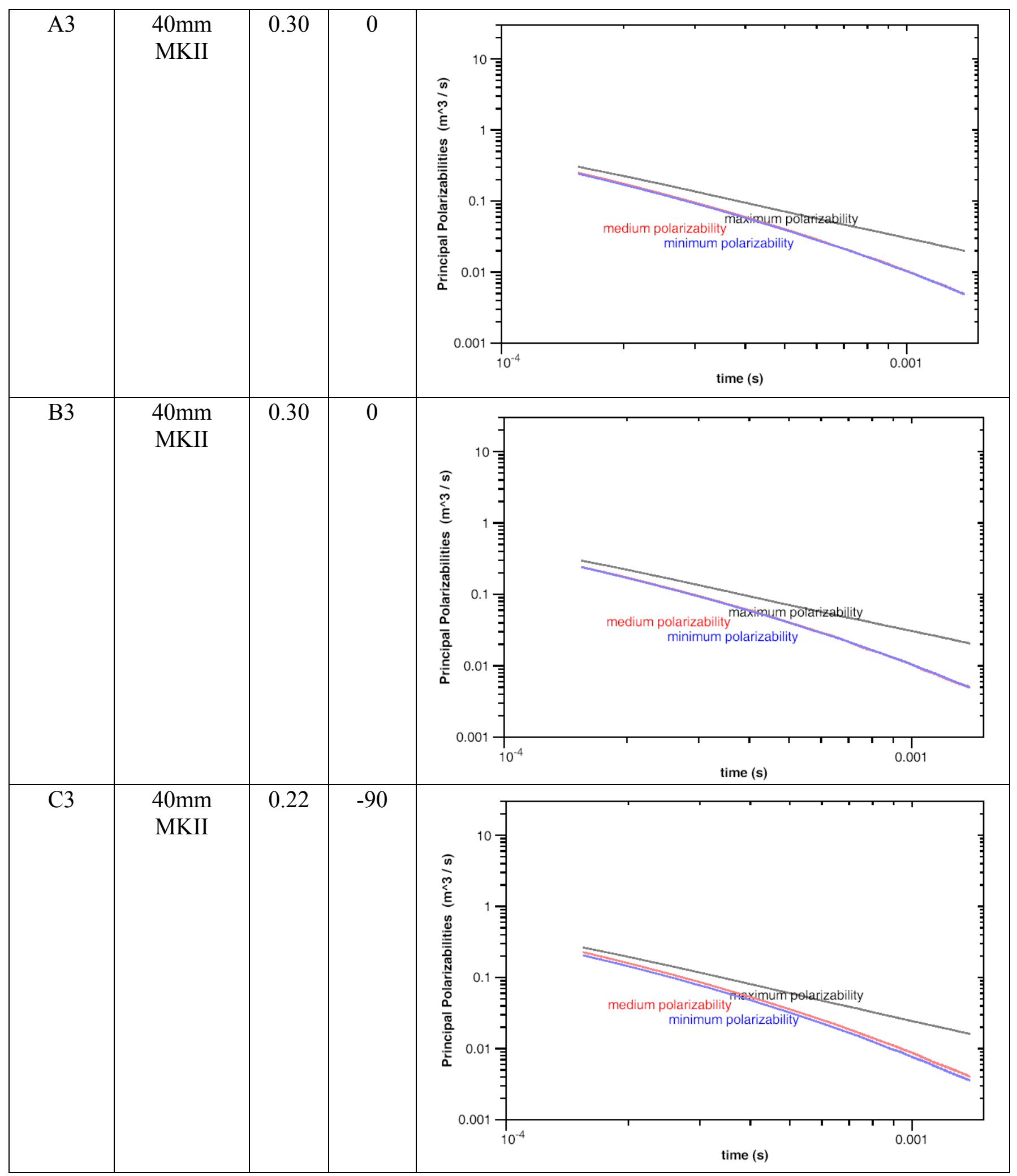




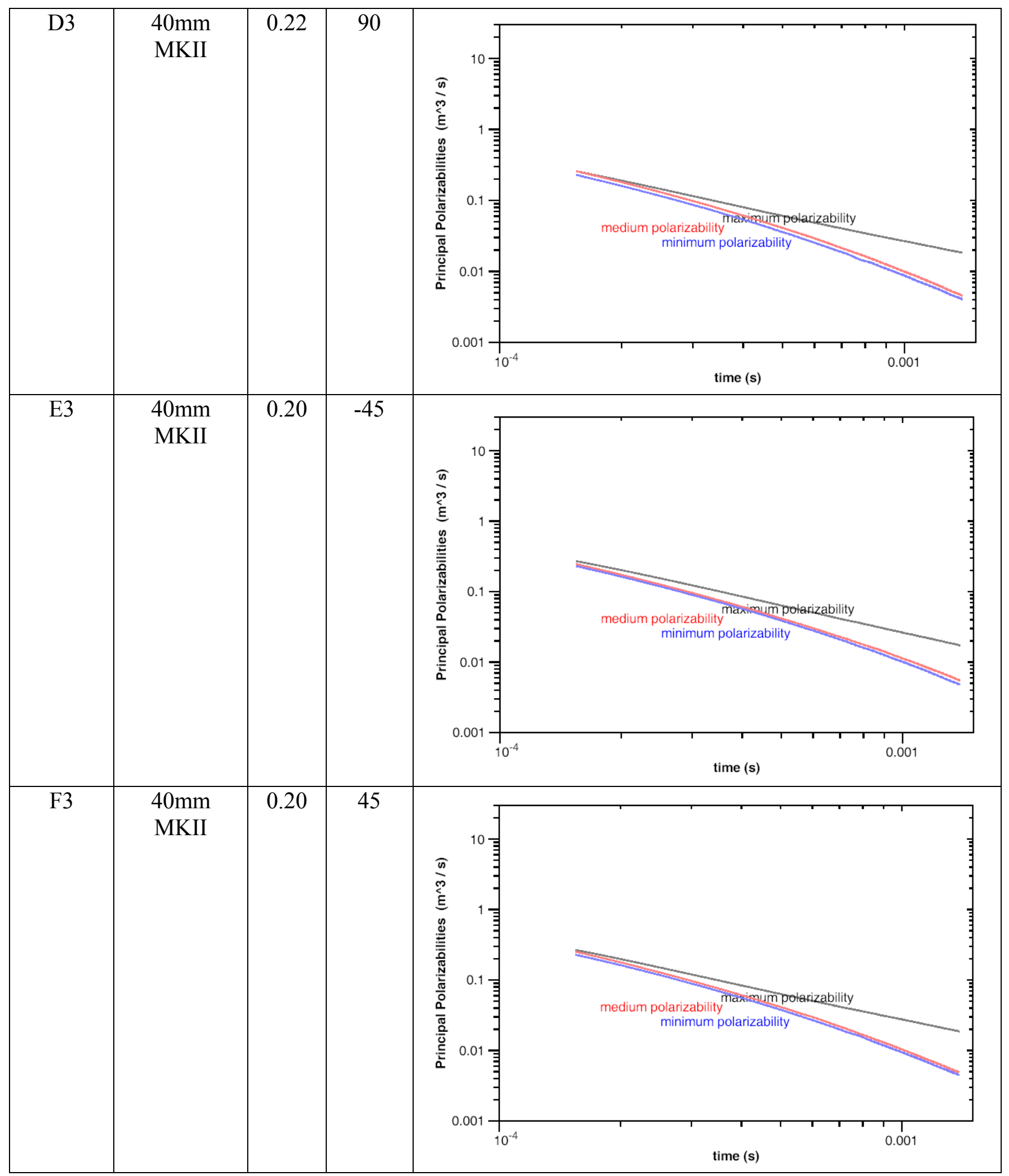




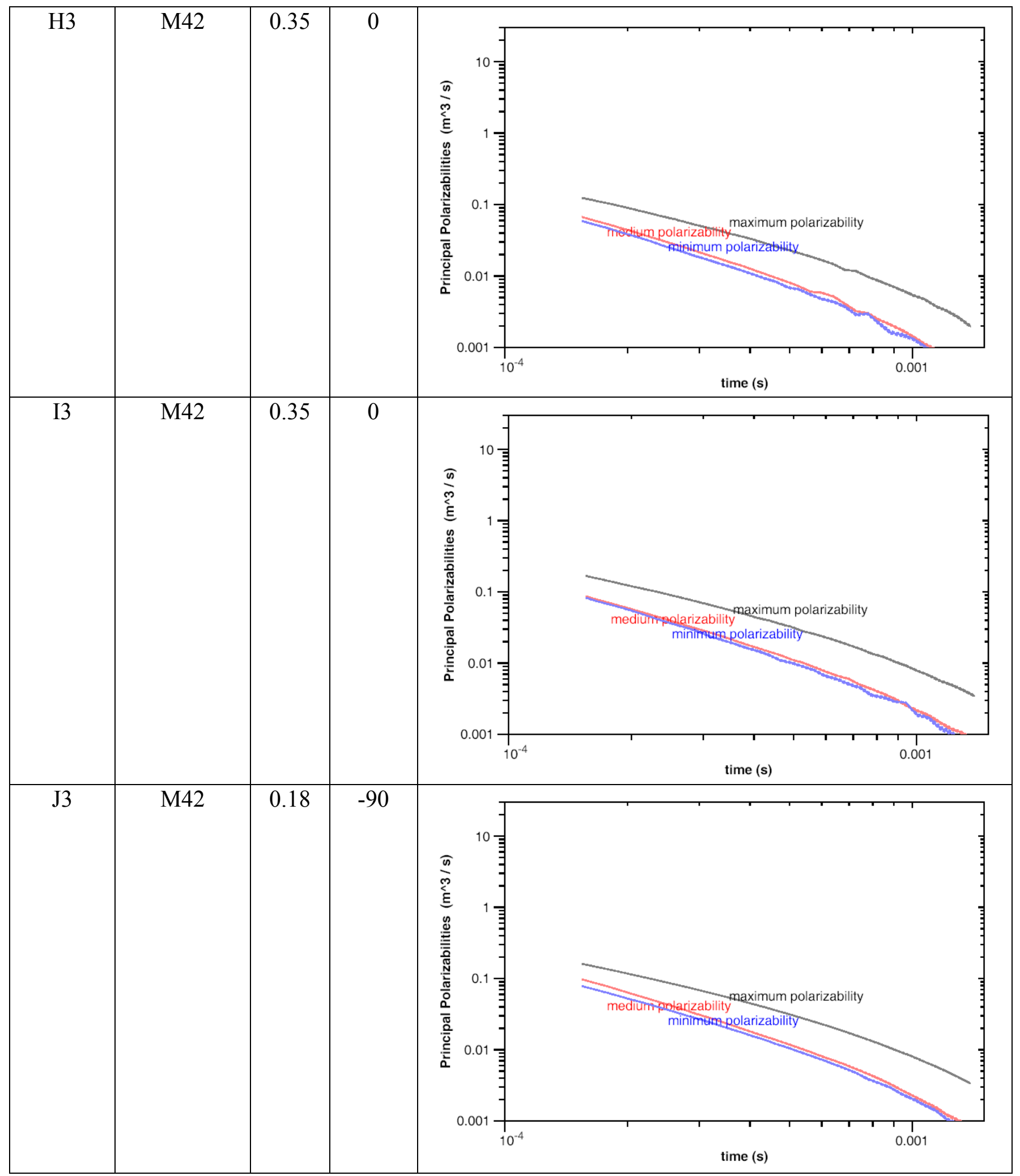




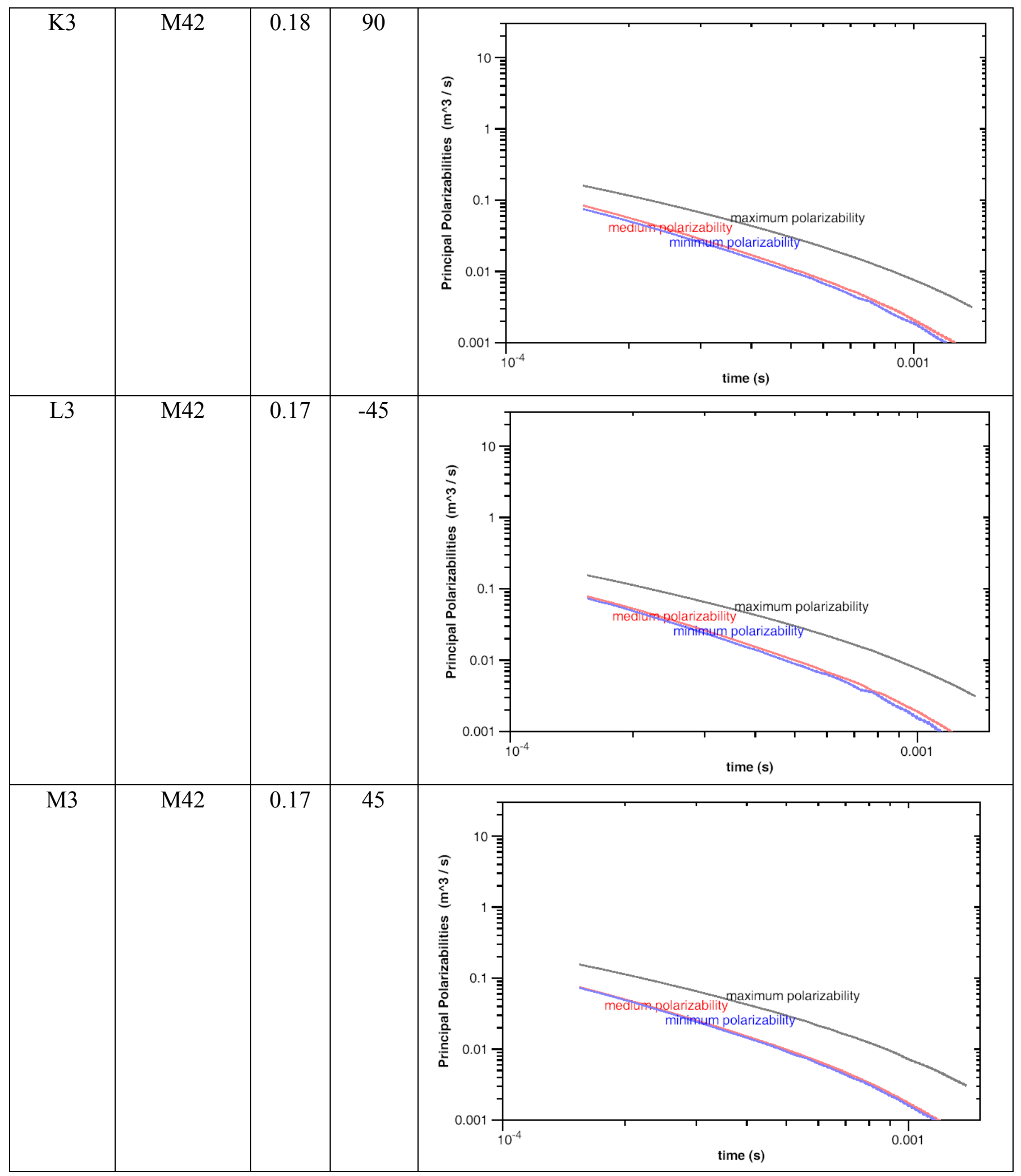




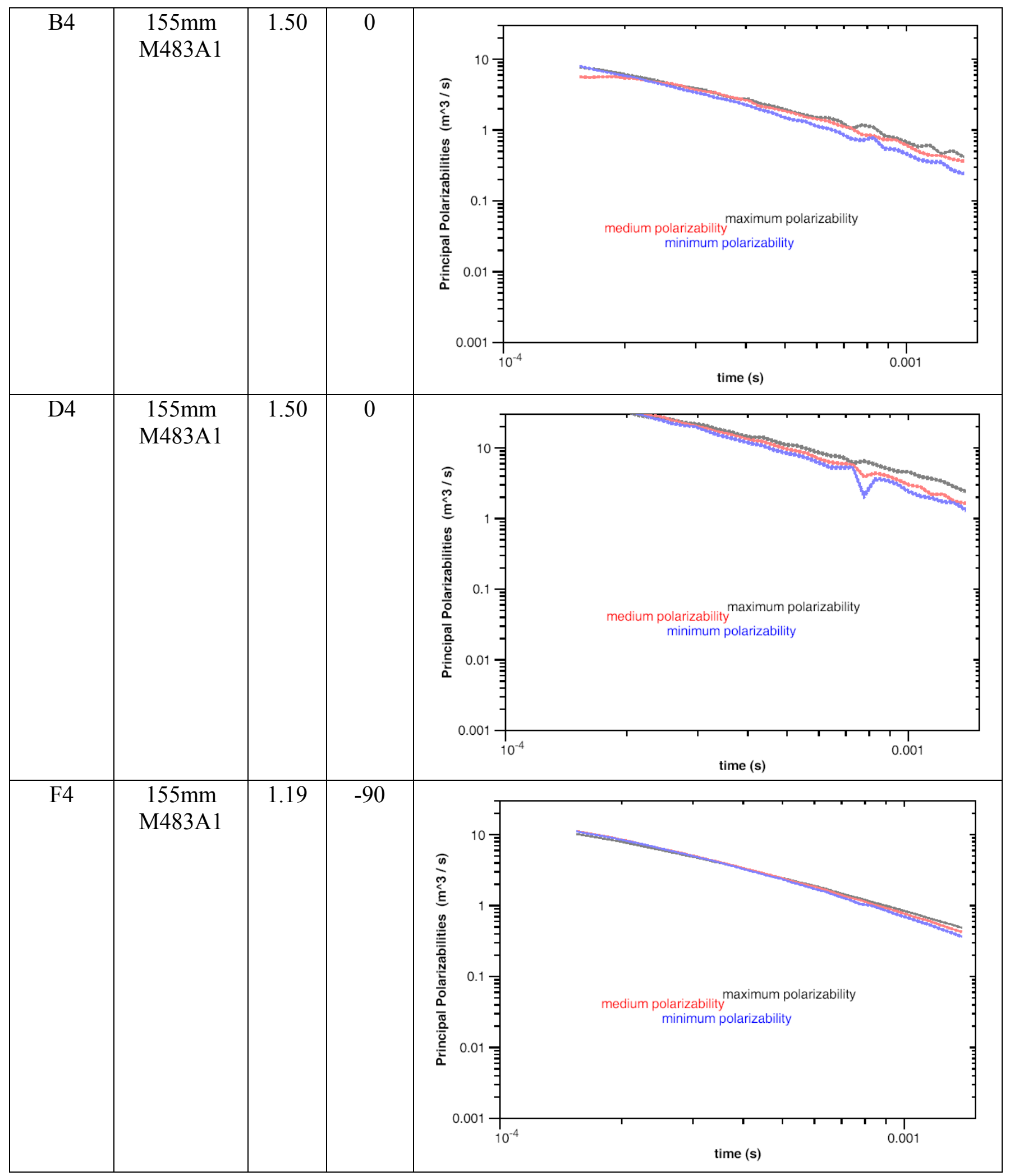




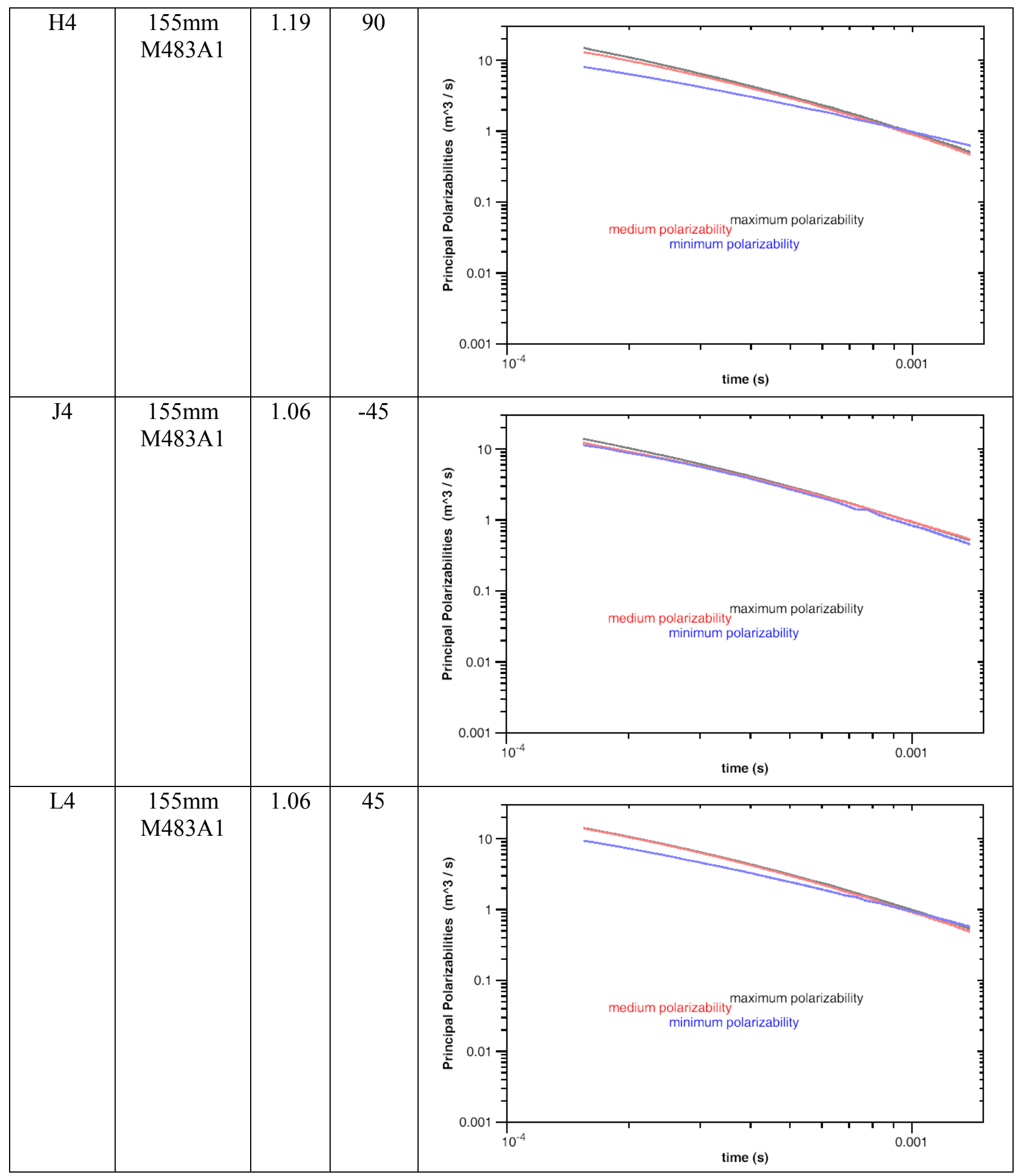




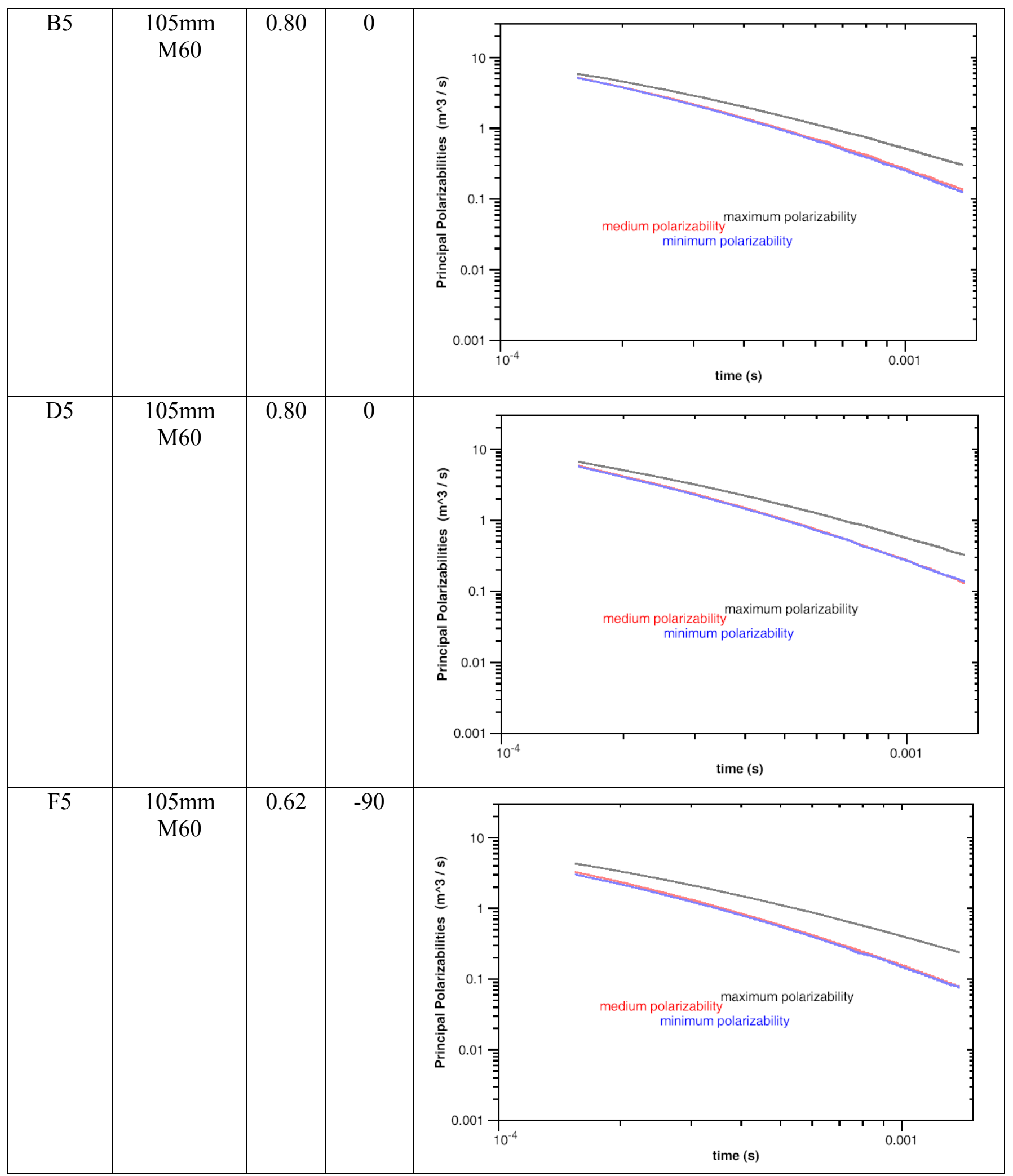




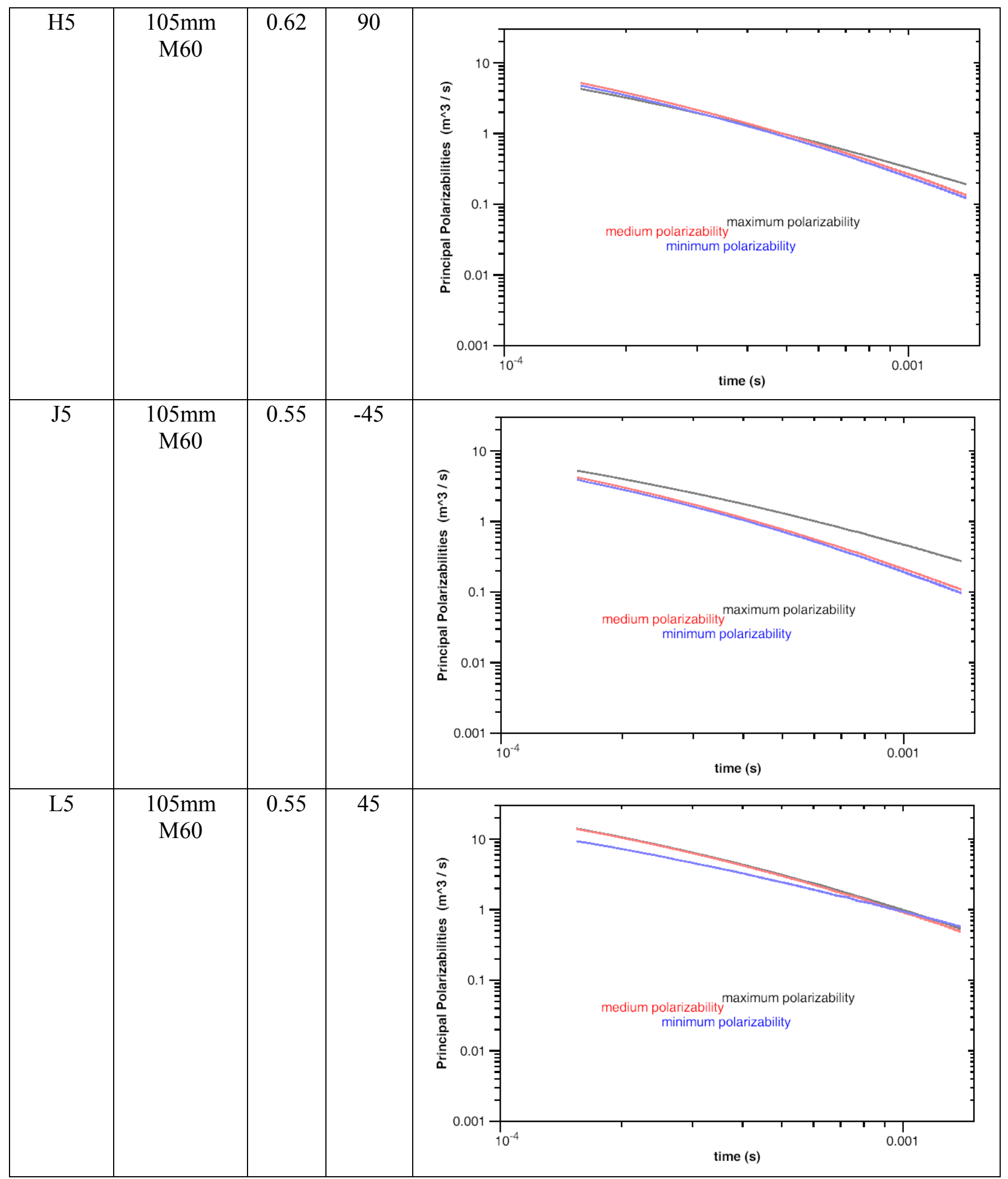




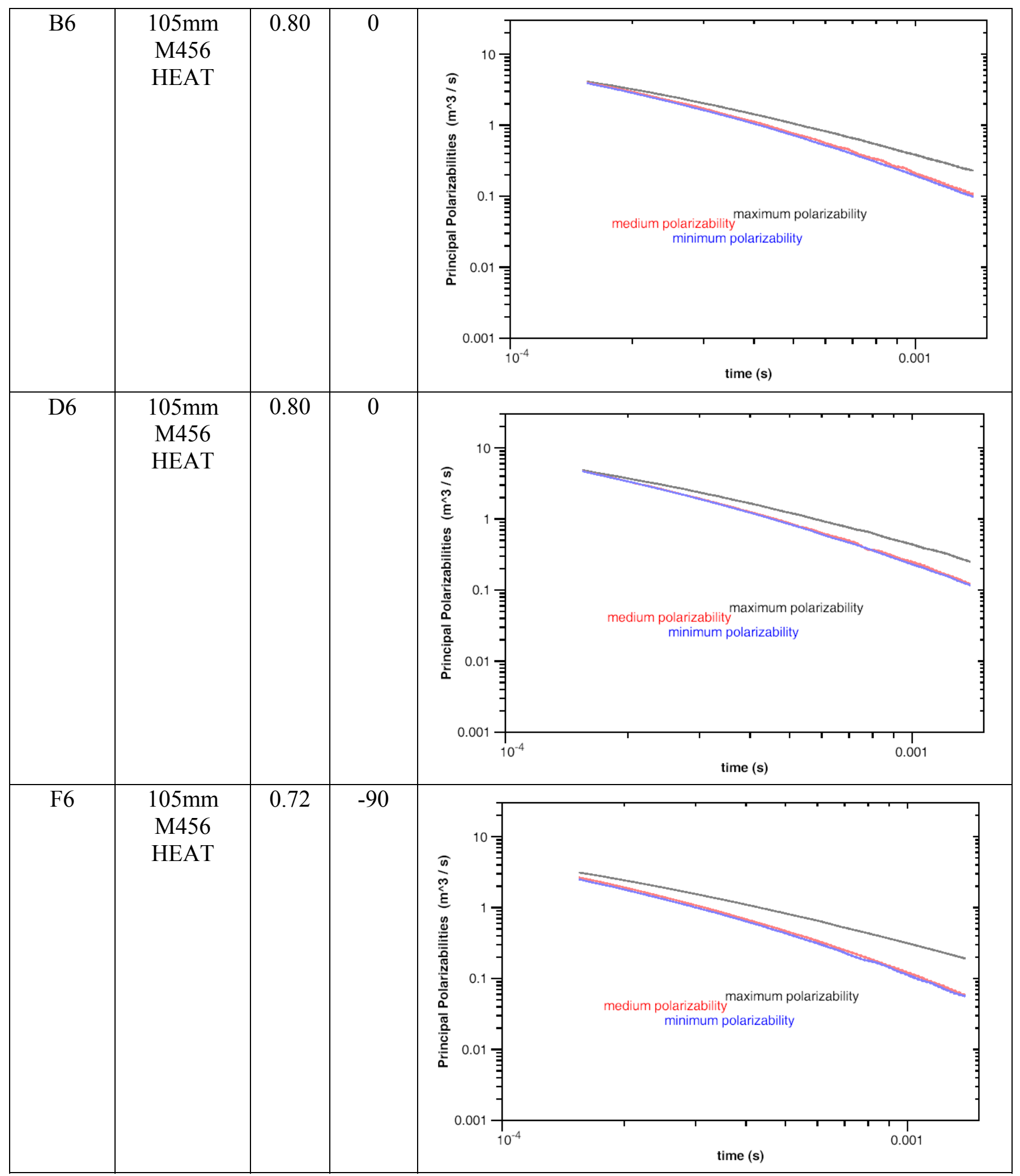




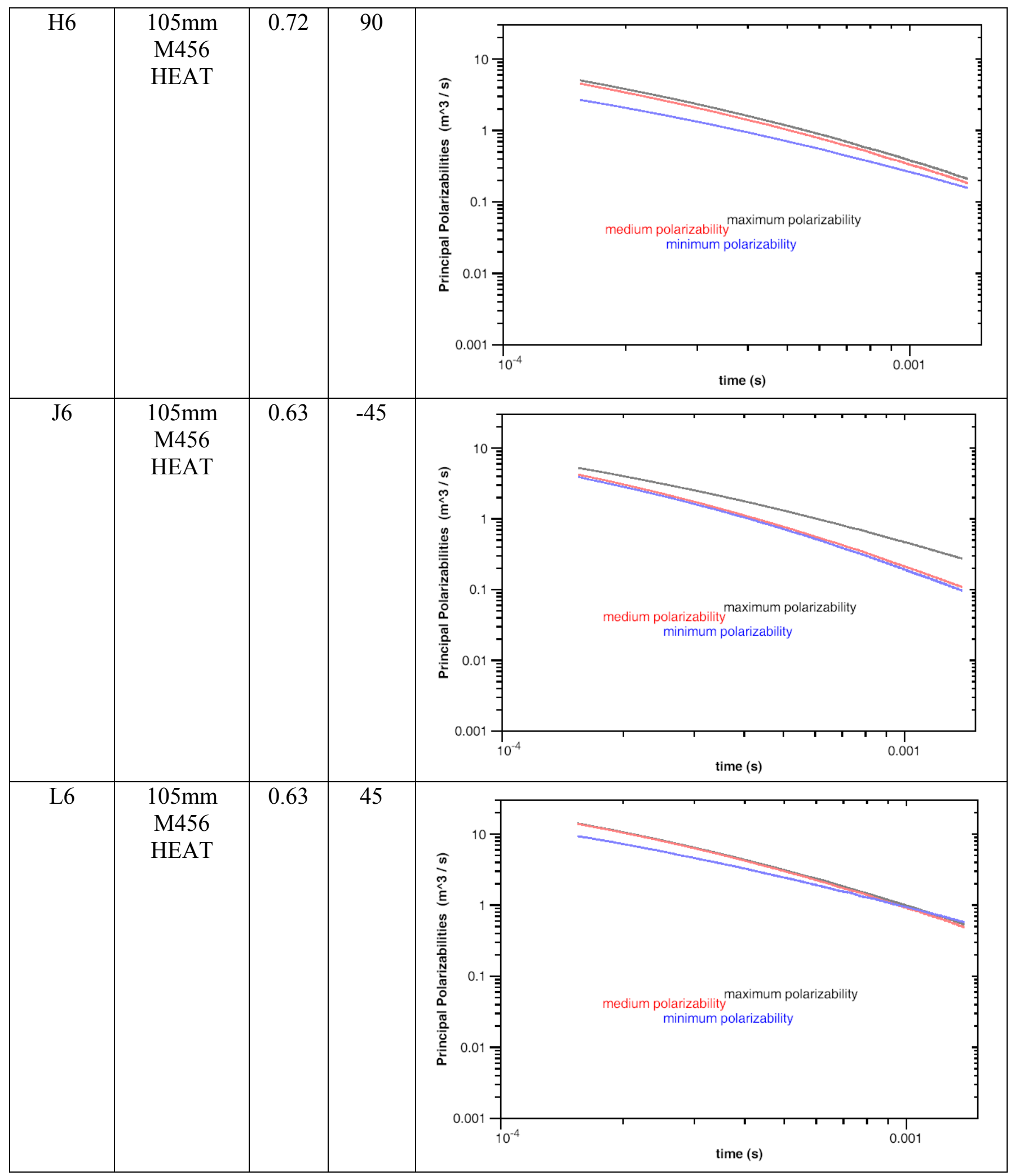




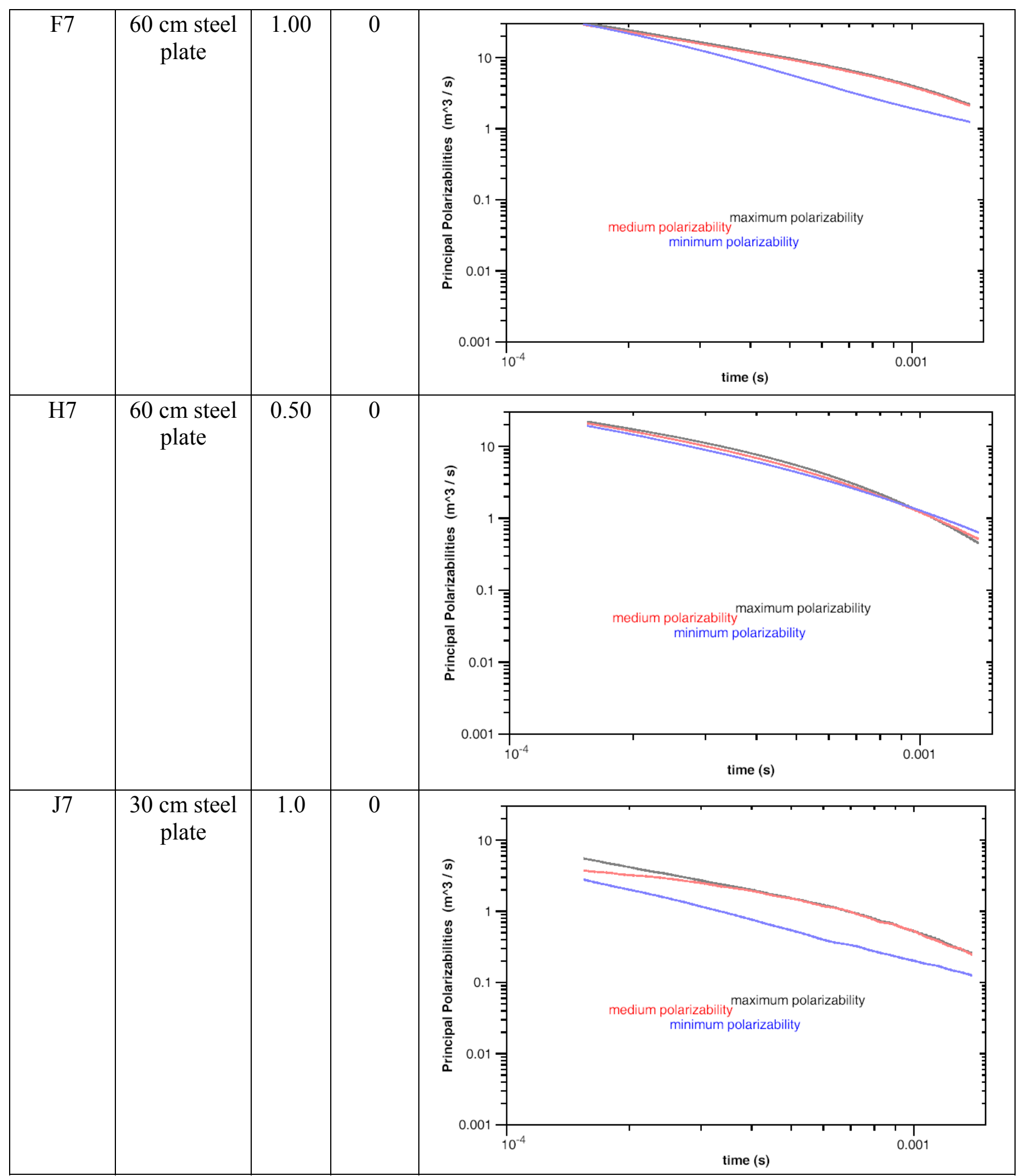




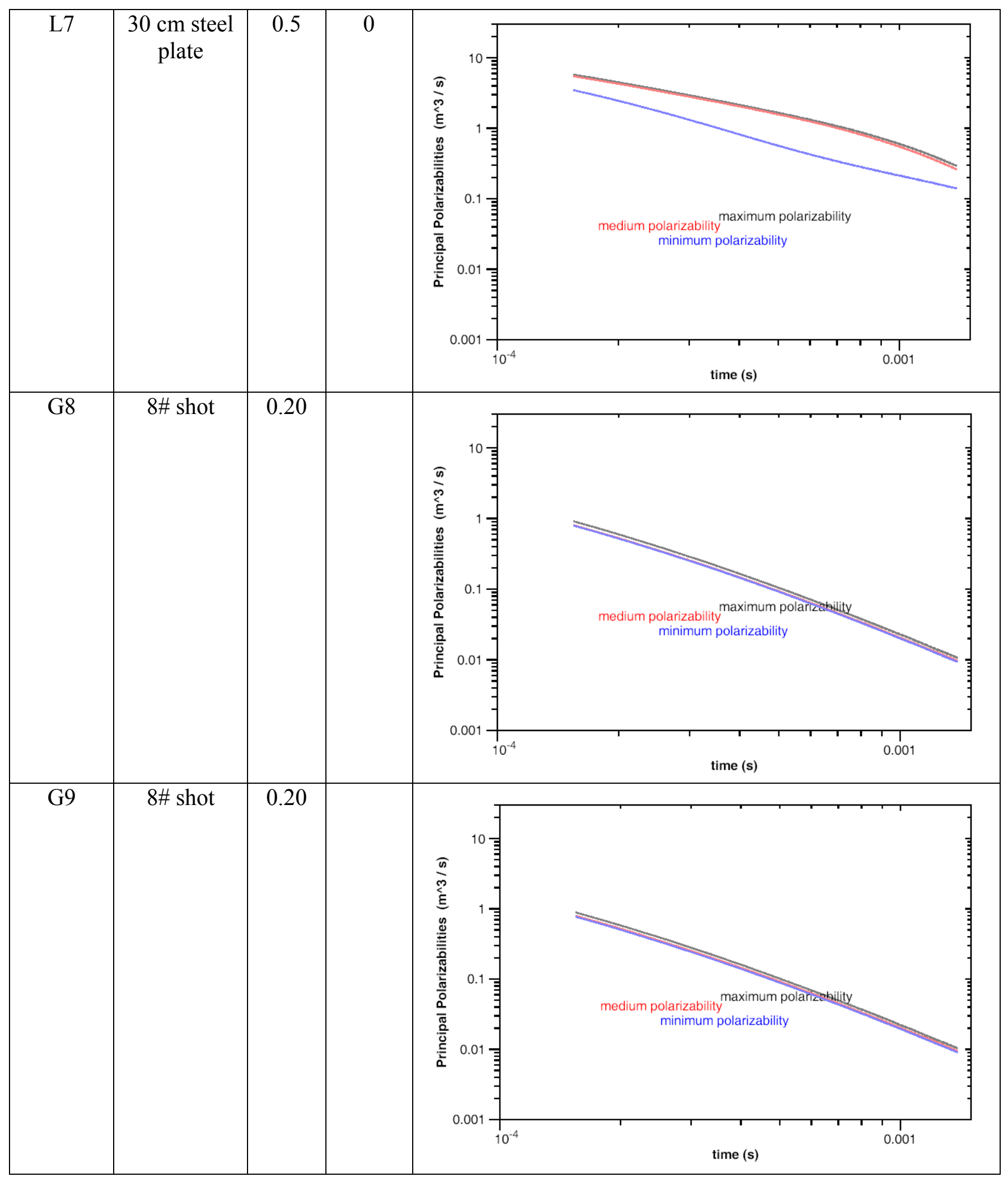




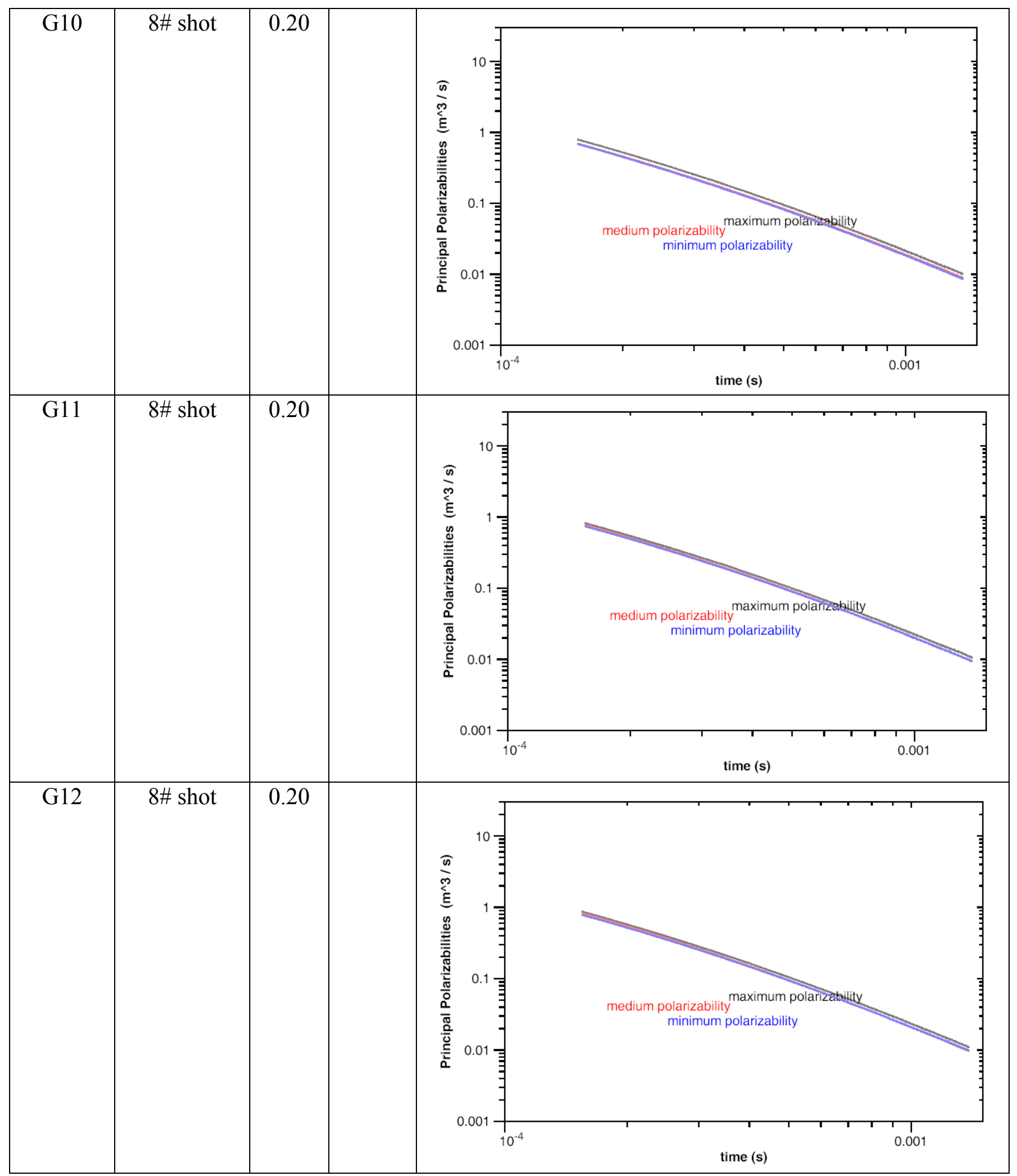




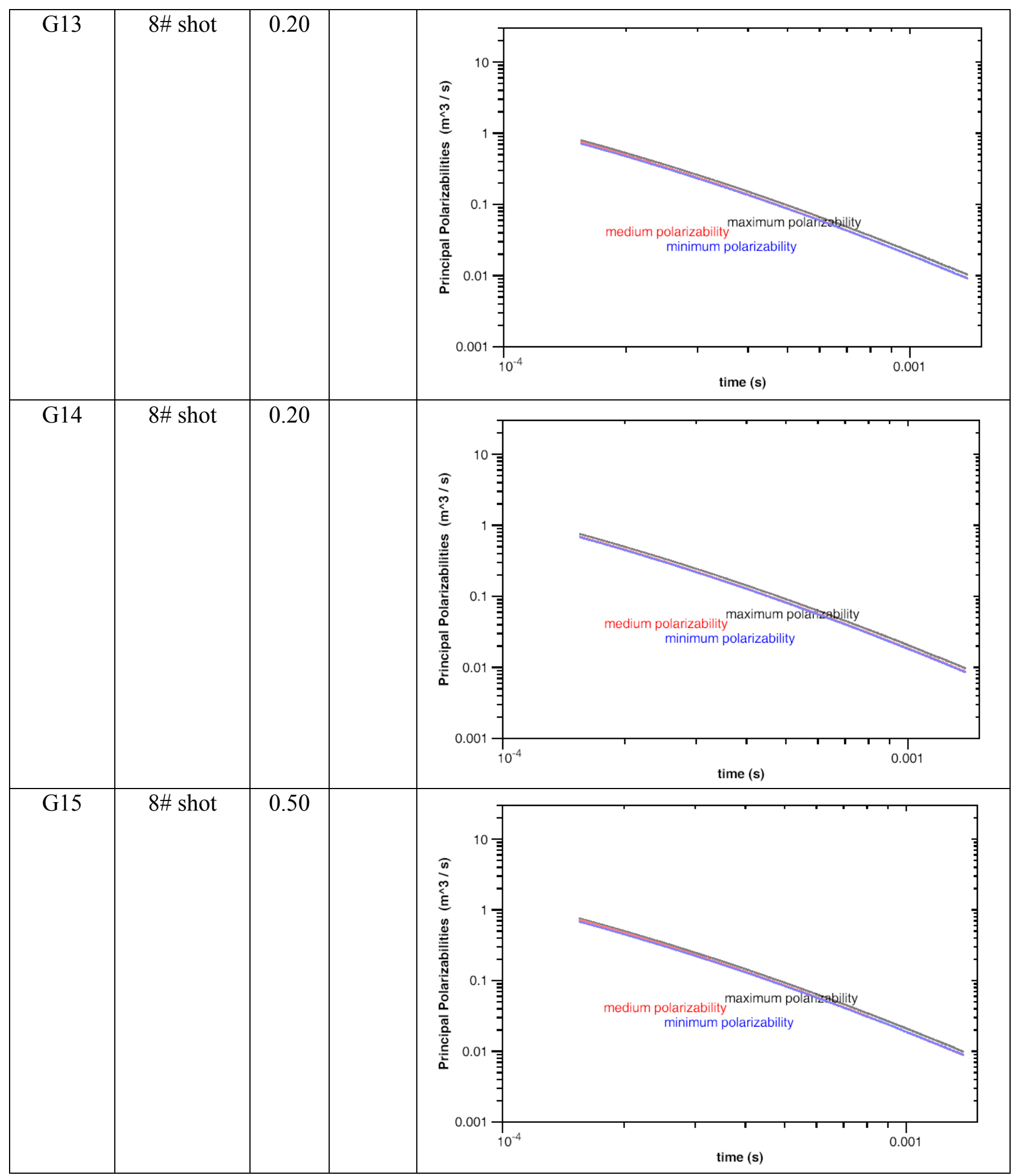




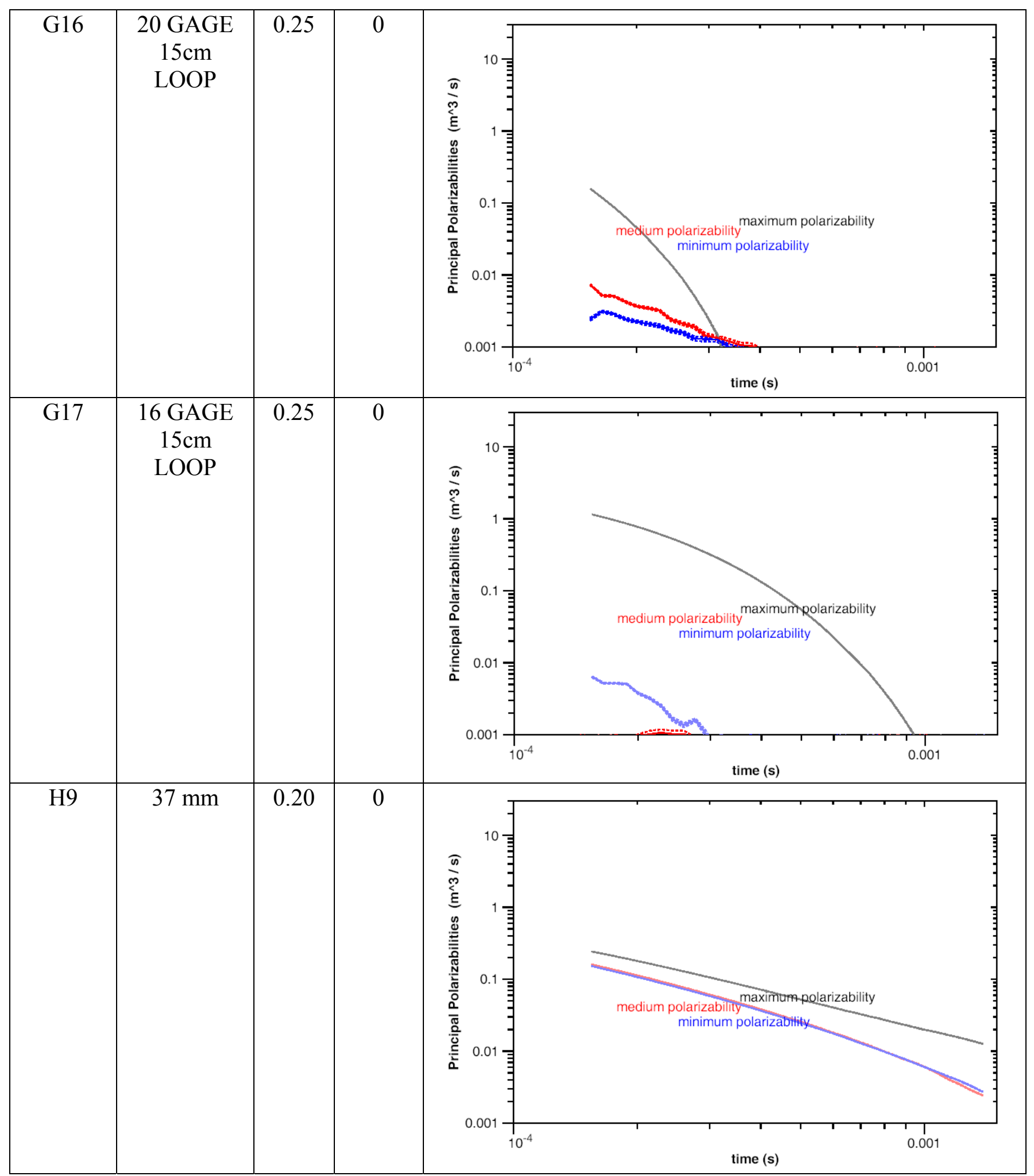




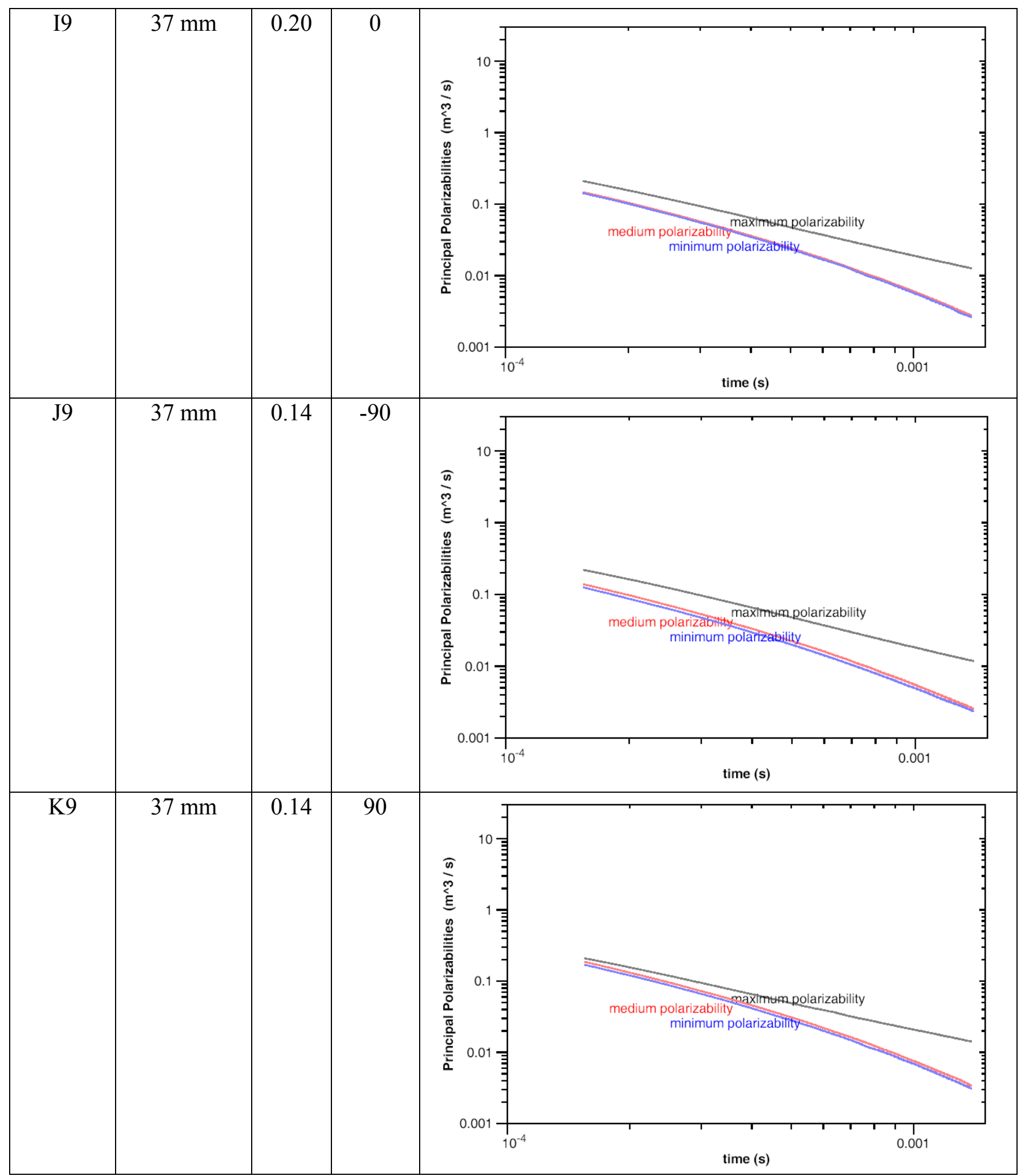




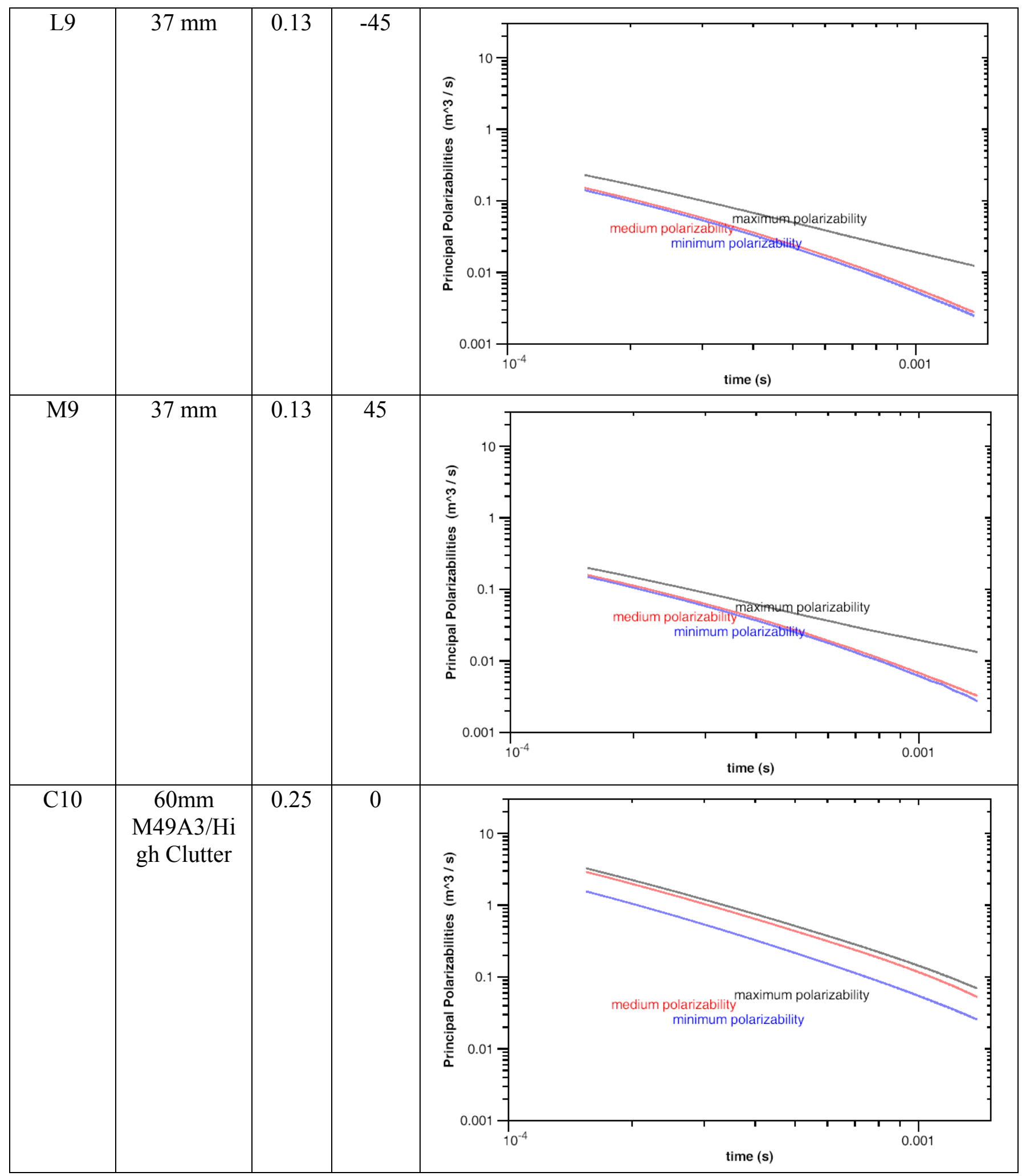




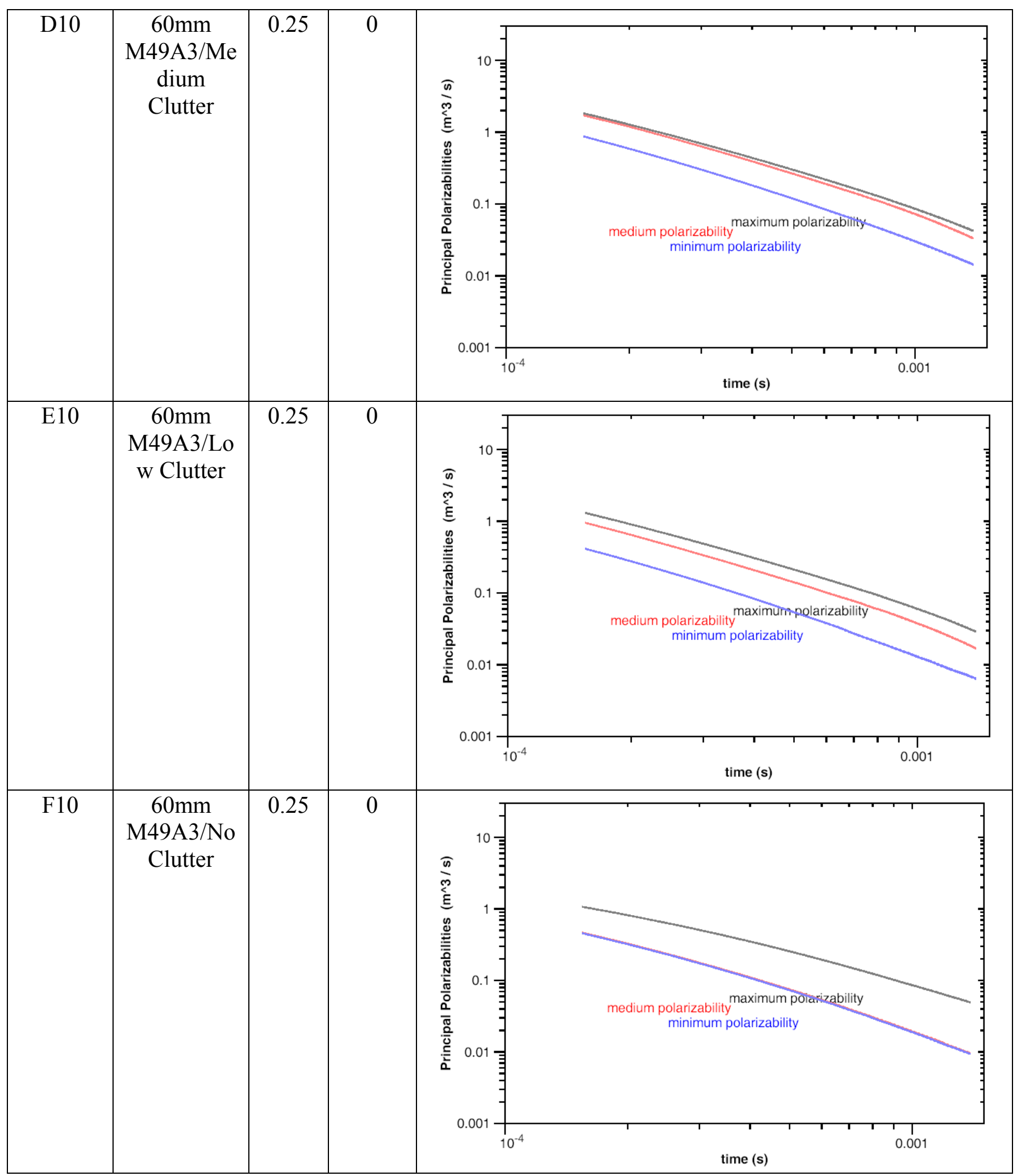




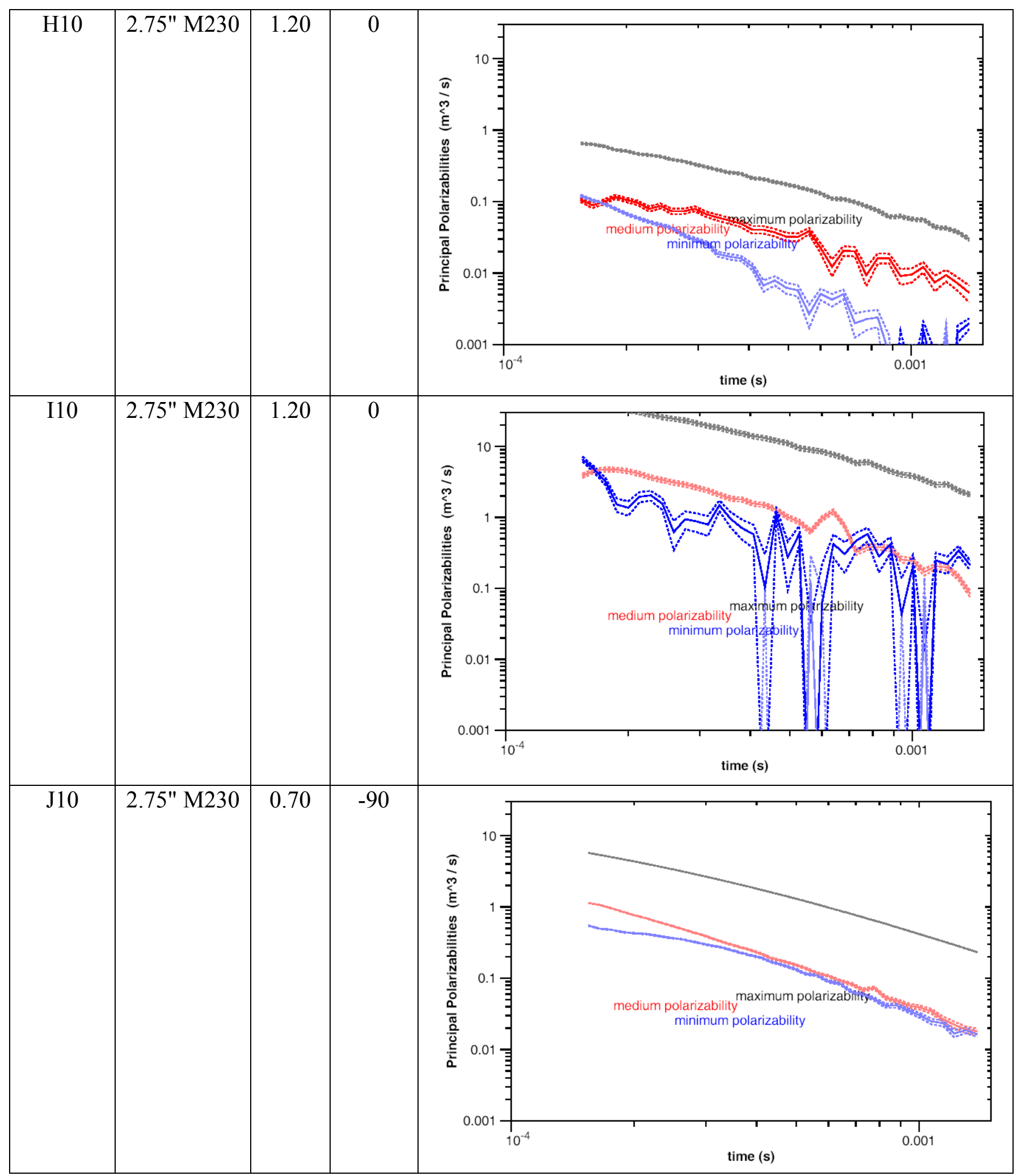




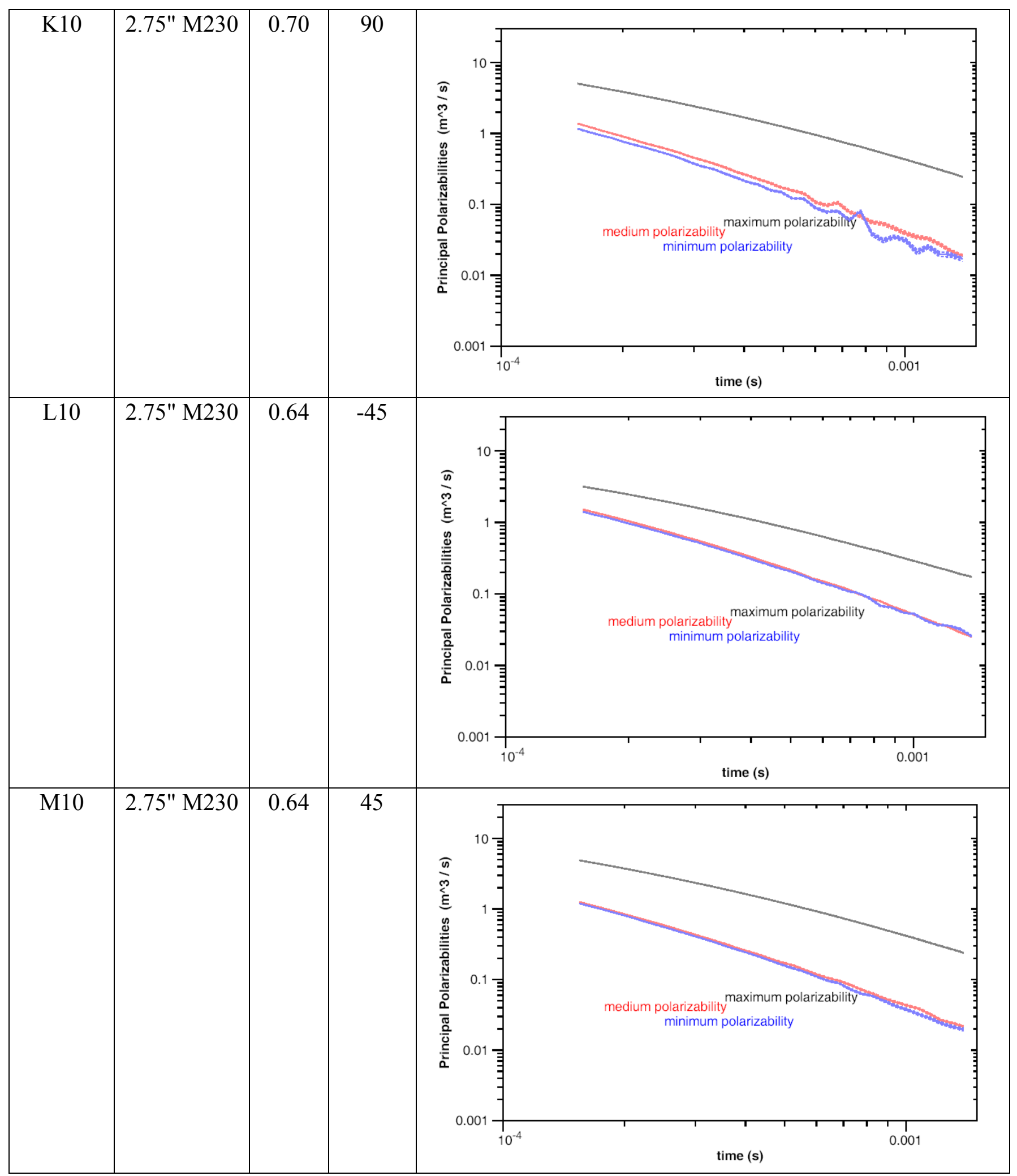




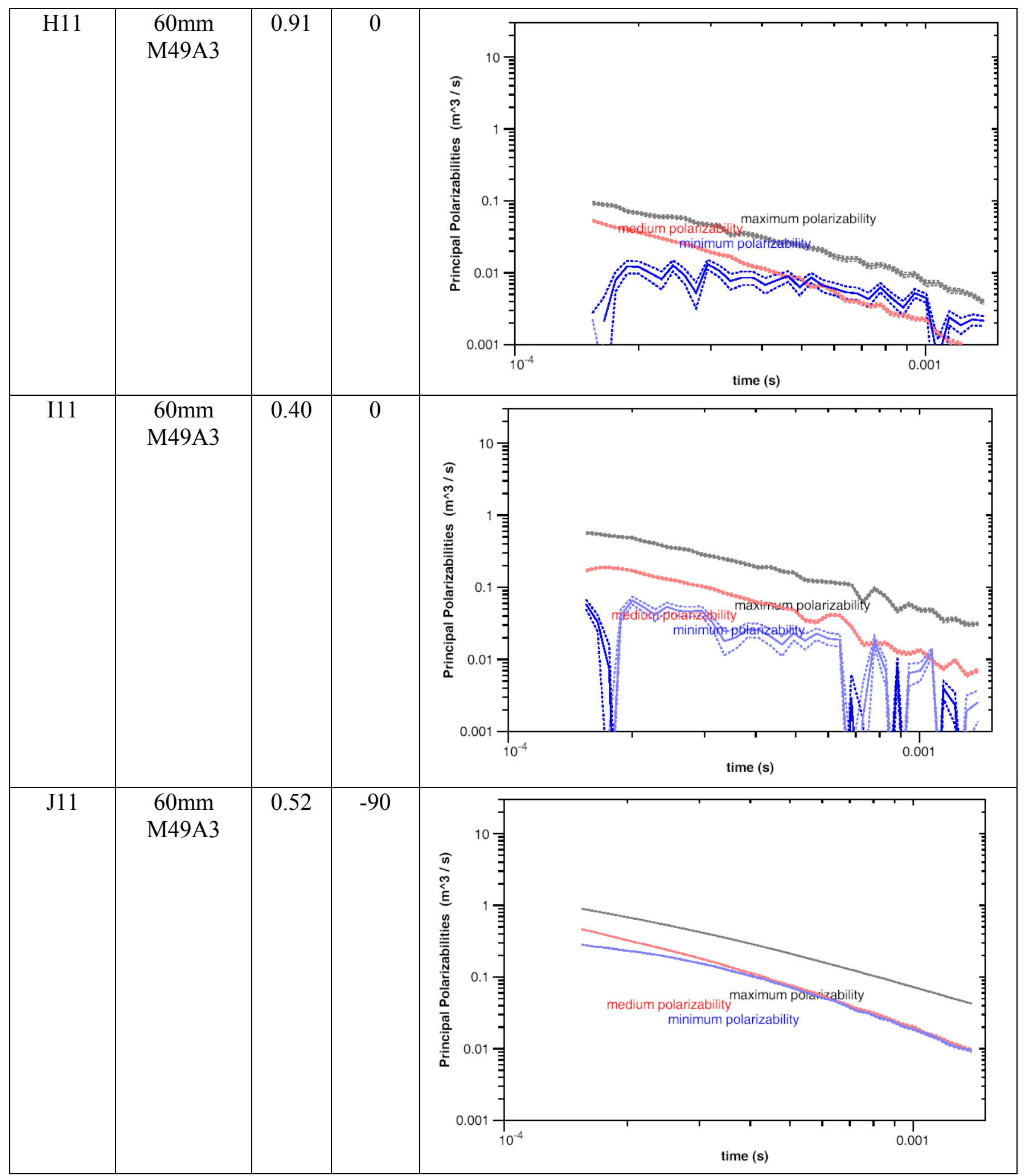




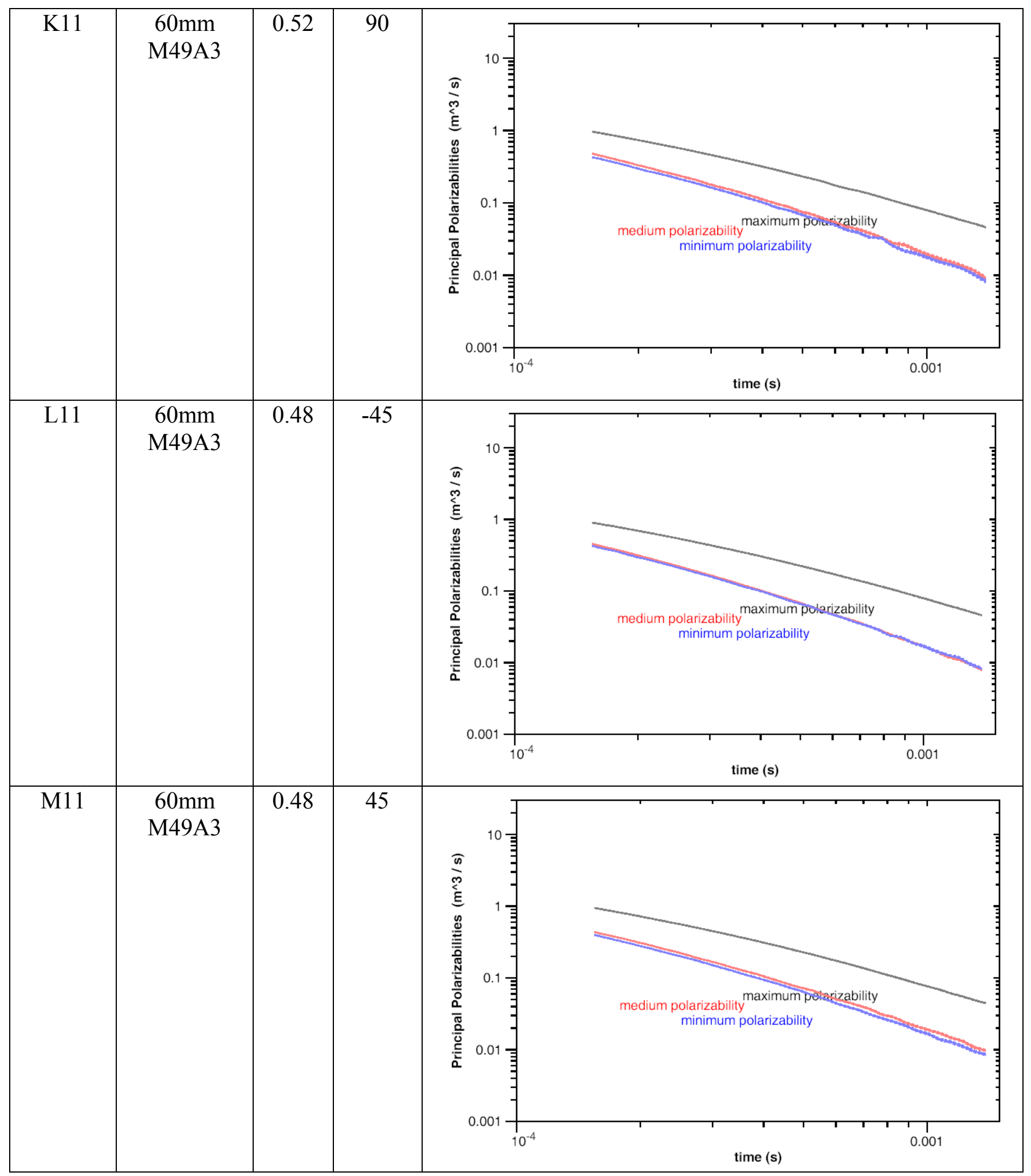




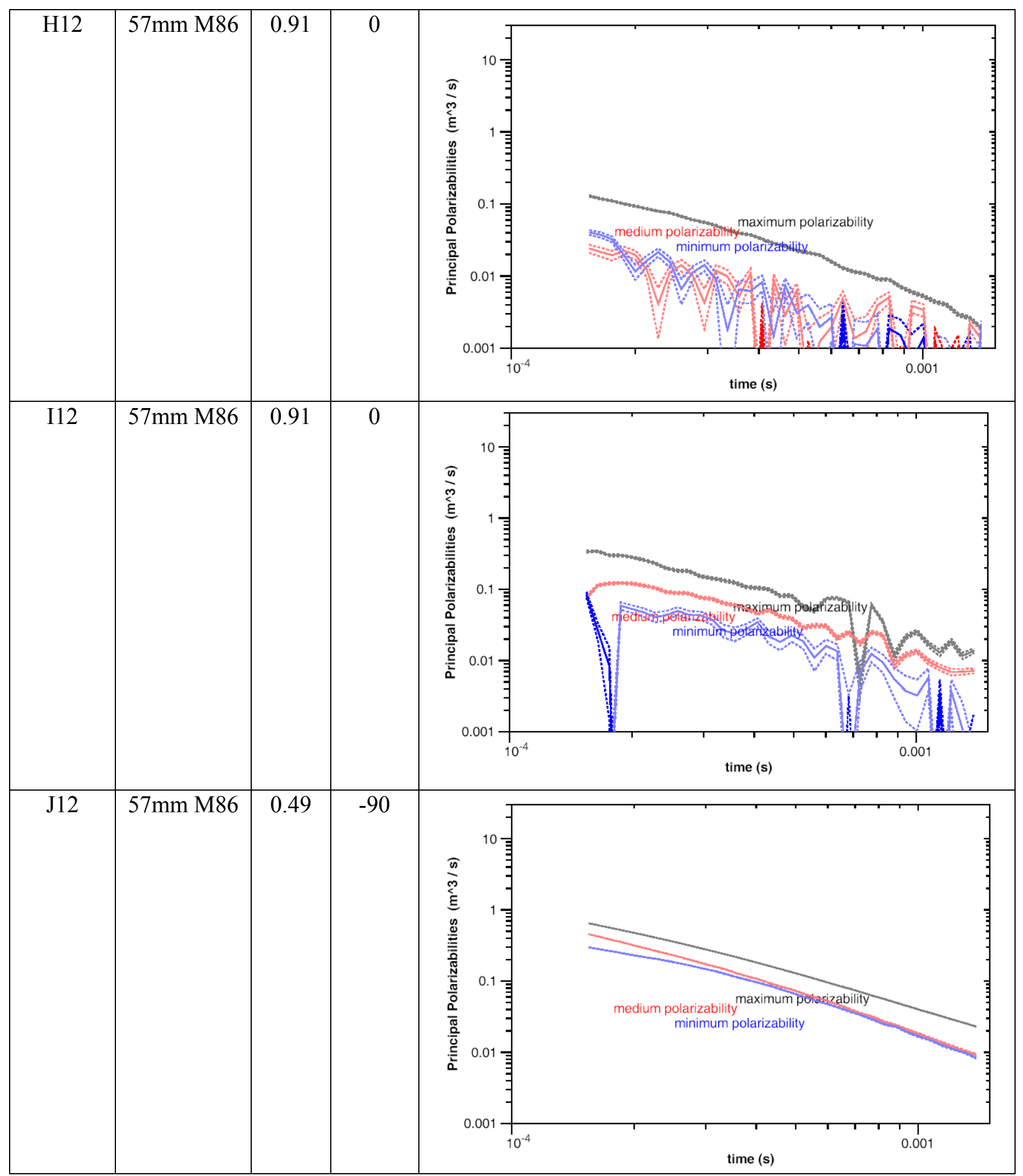




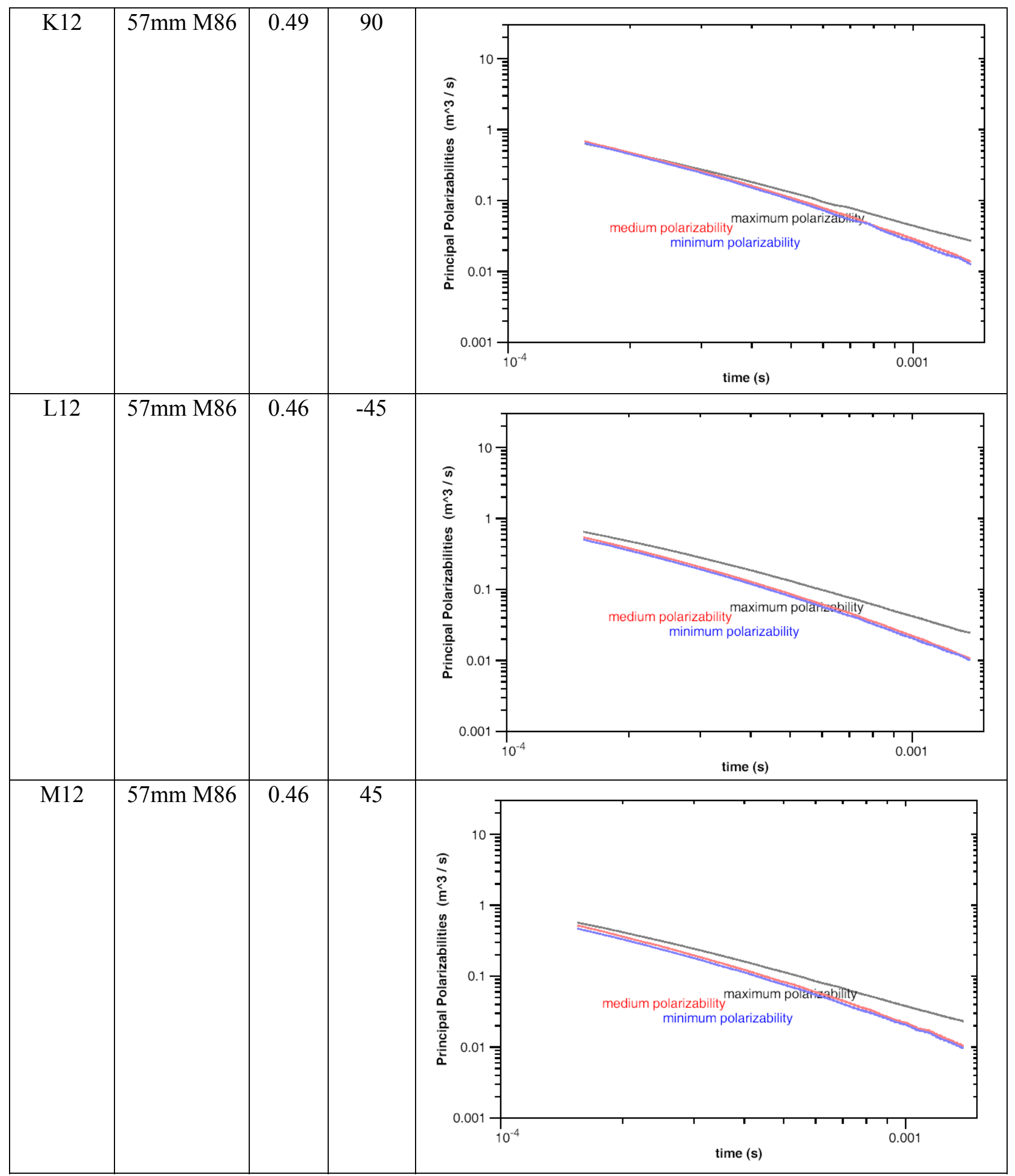




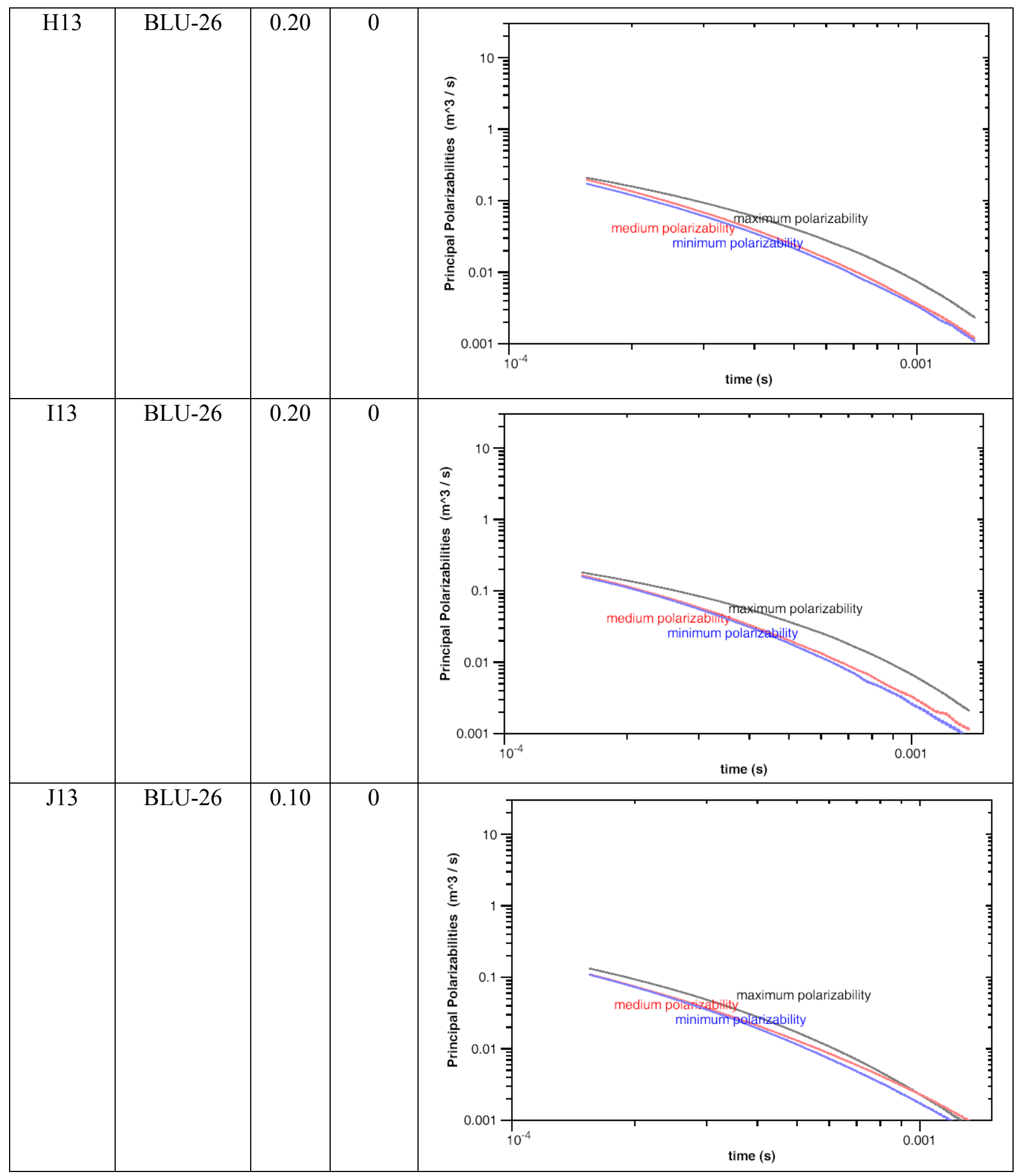




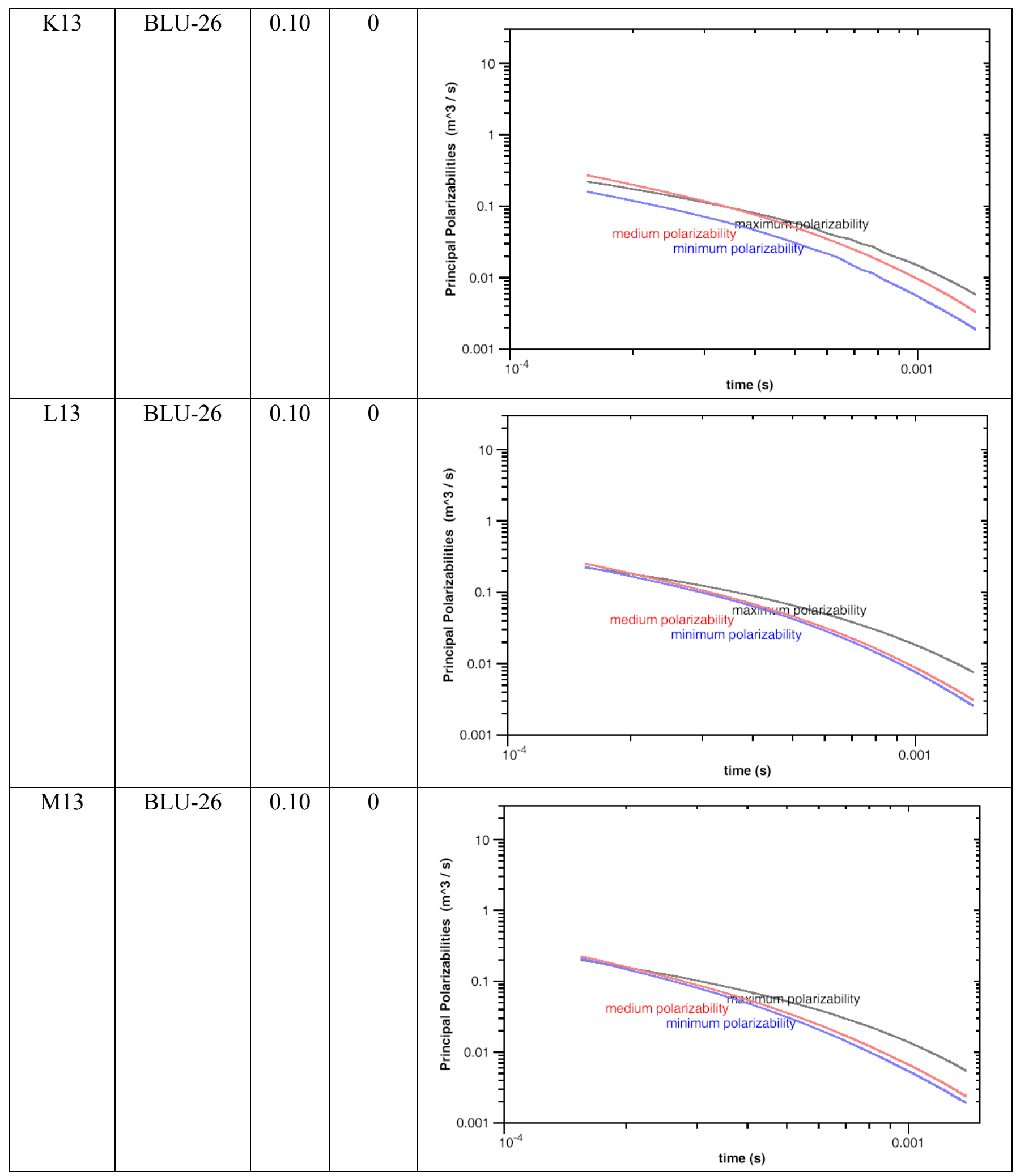




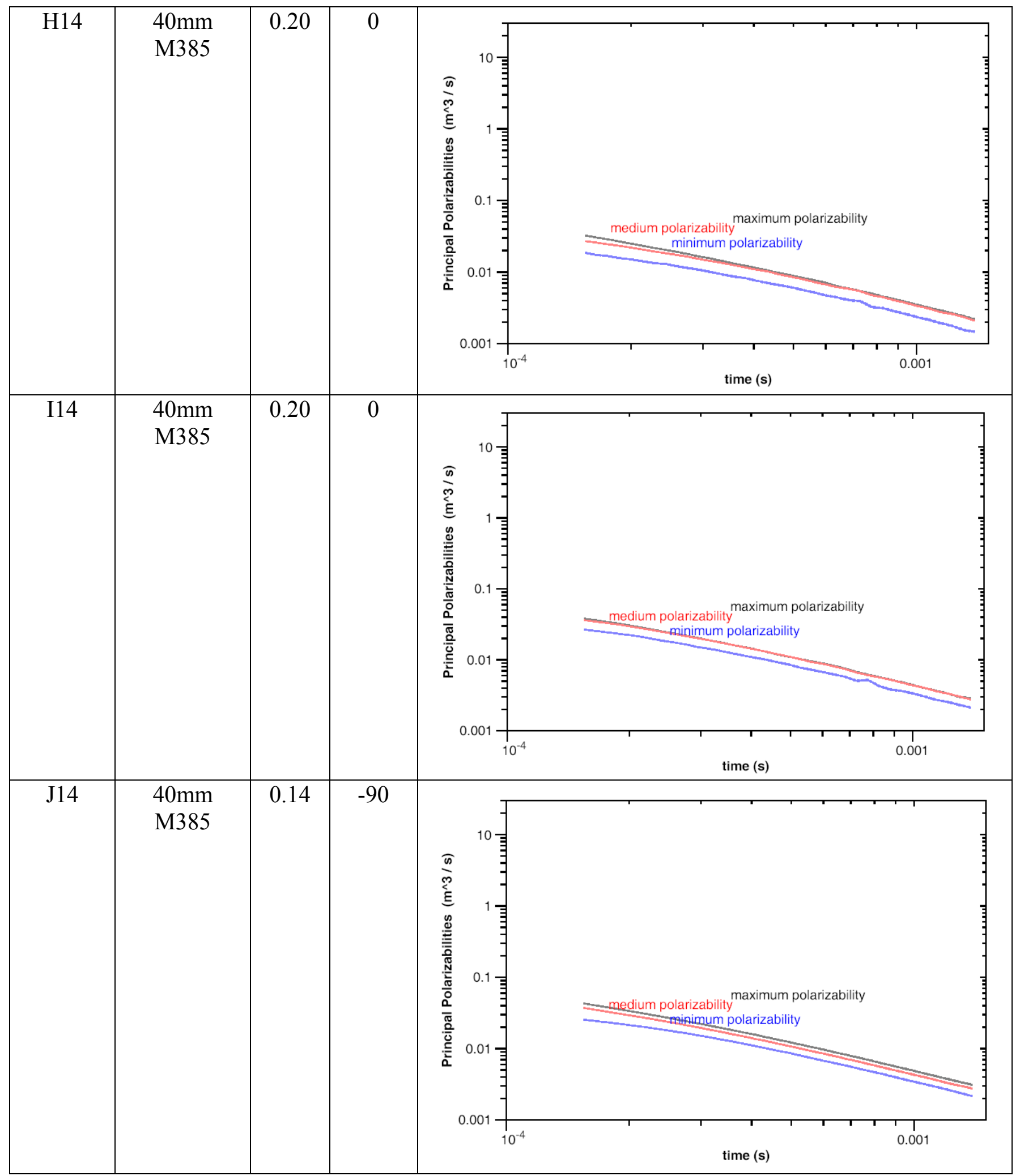




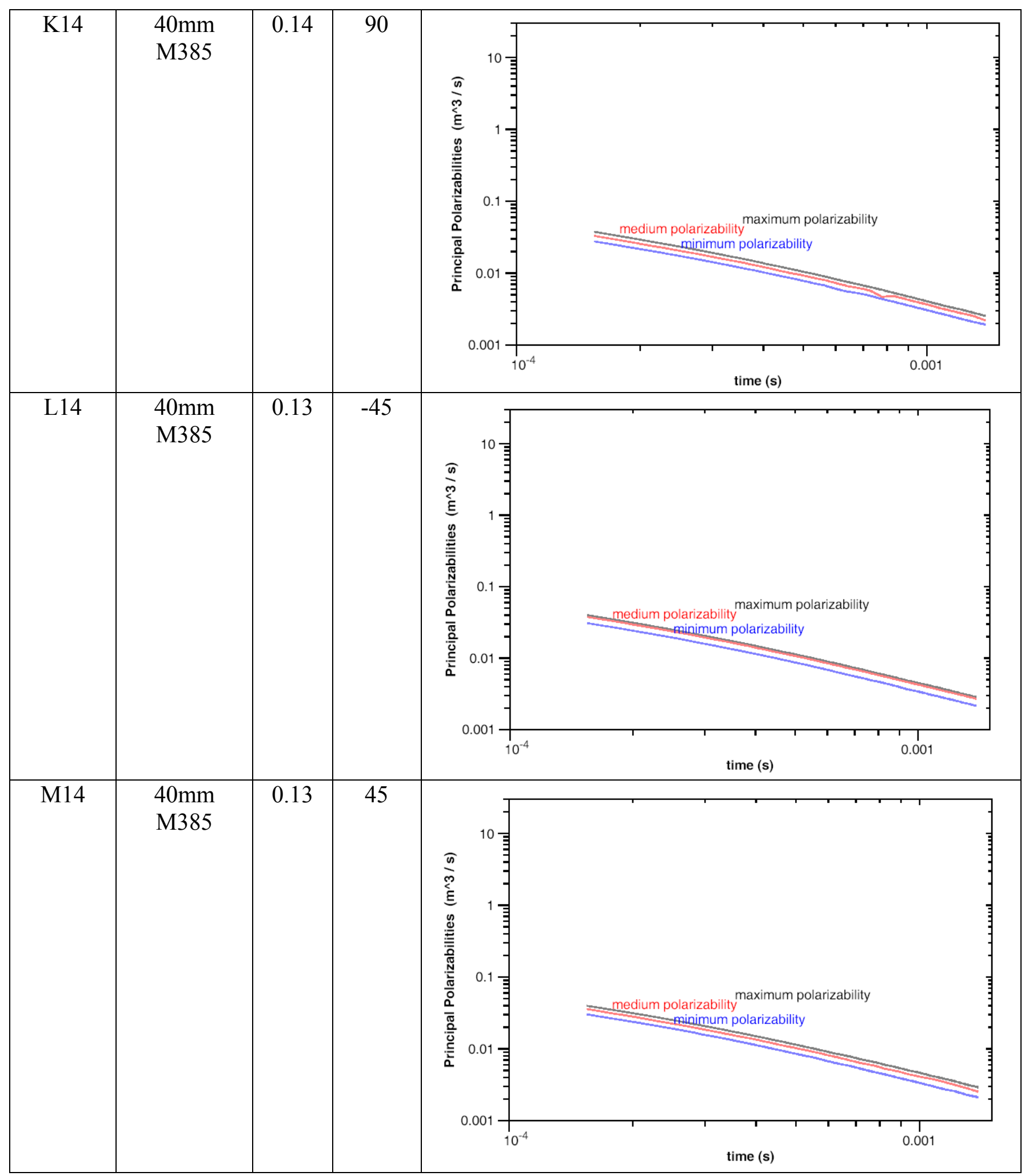




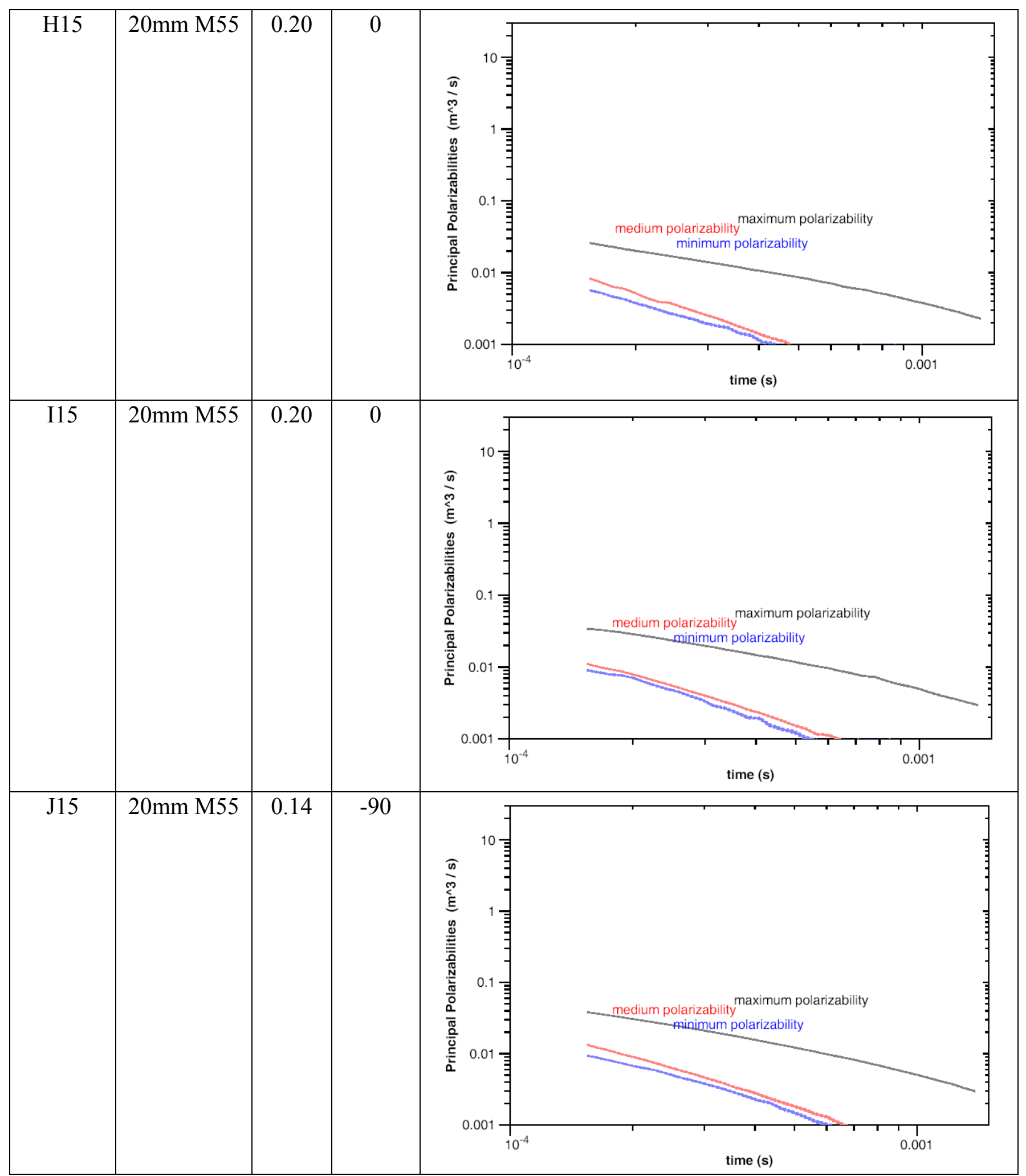




\begin{tabular}{|c|c|c|c|c|c|}
\hline K15 & 20mm M55 & 0.14 & 90 & 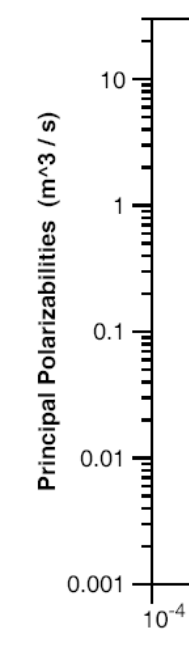 & medium polarizability maximum polarizability \\
\hline L15 & 20mm M55 & 0.13 & -45 & 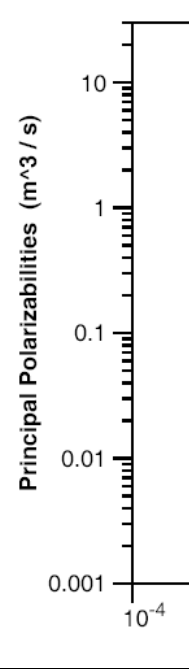 & medium polarizability maximum polarizability \\
\hline M15 & 20mm M55 & 0.13 & 45 & 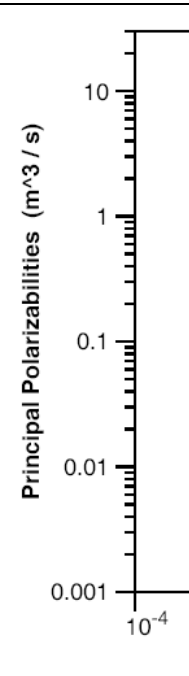 & medium polarizability maximum polarizability \\
\hline
\end{tabular}




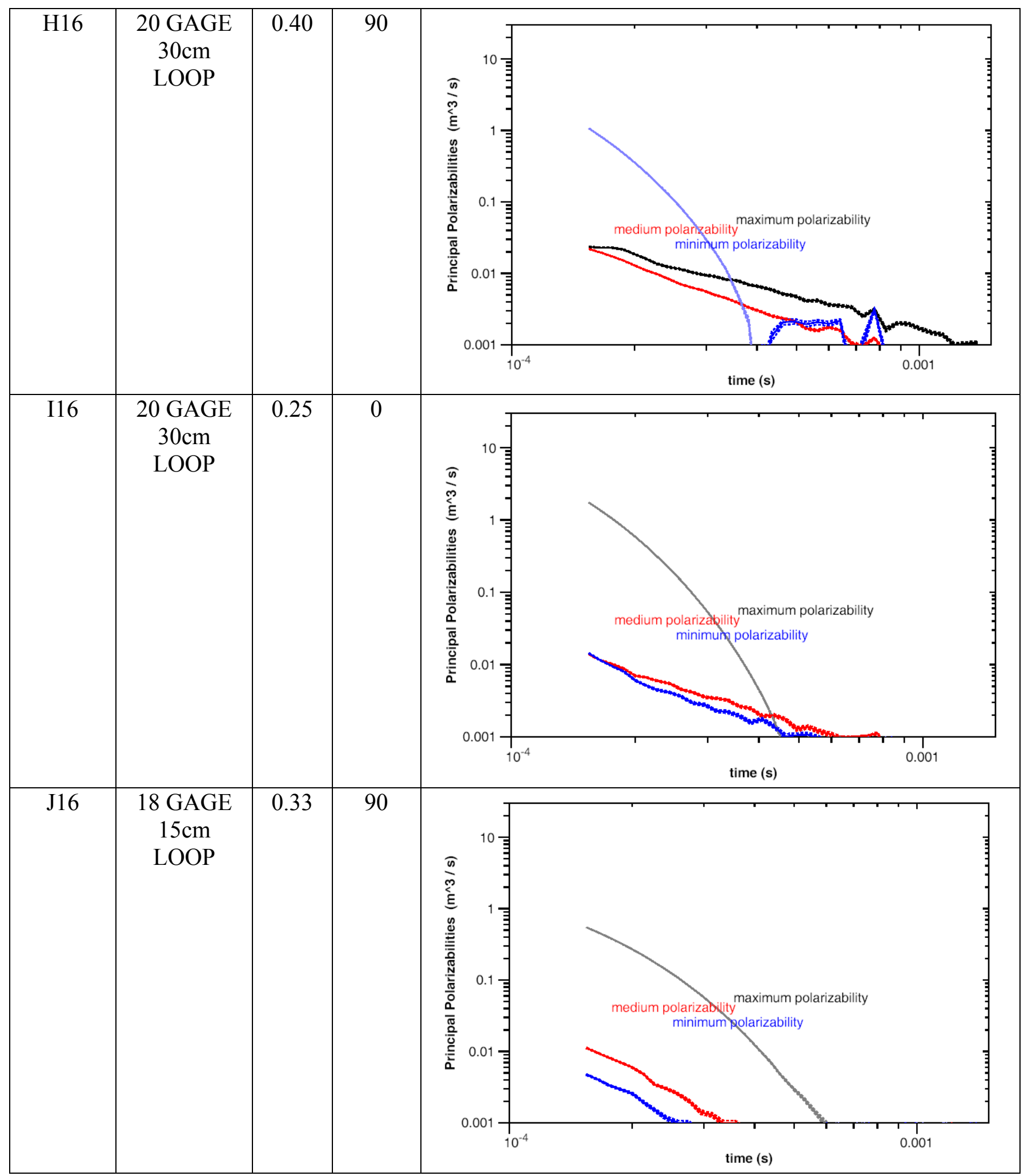




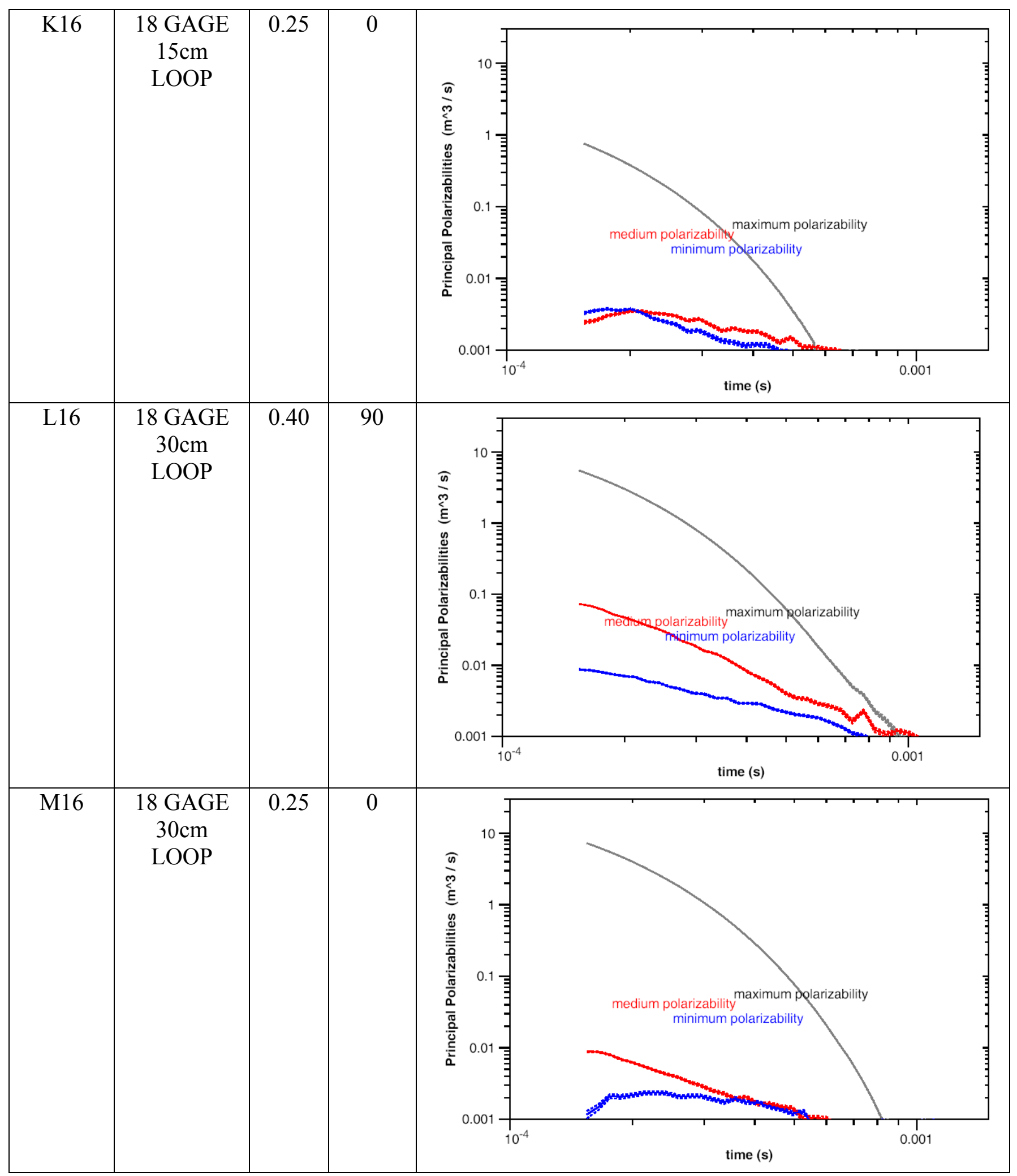




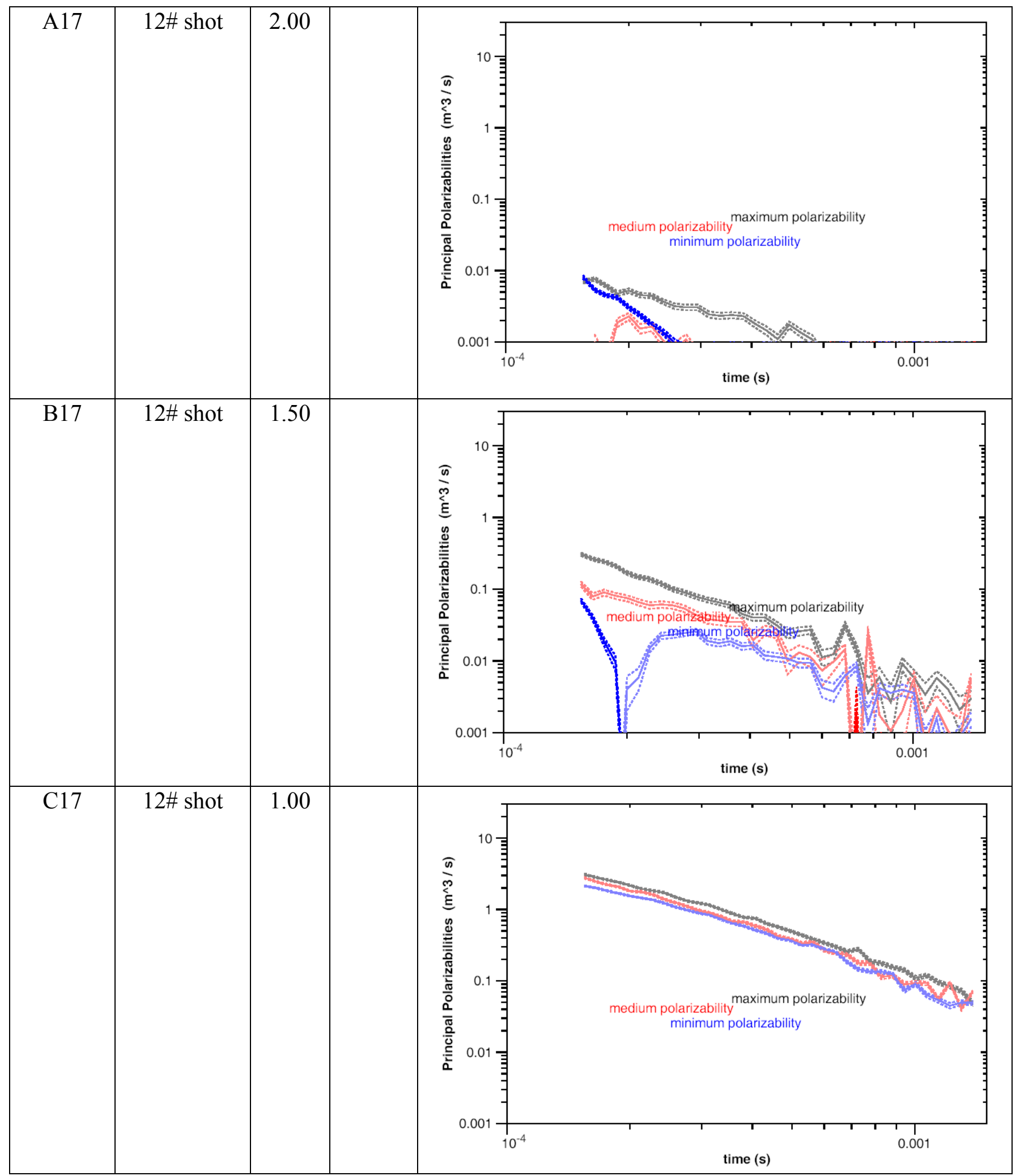




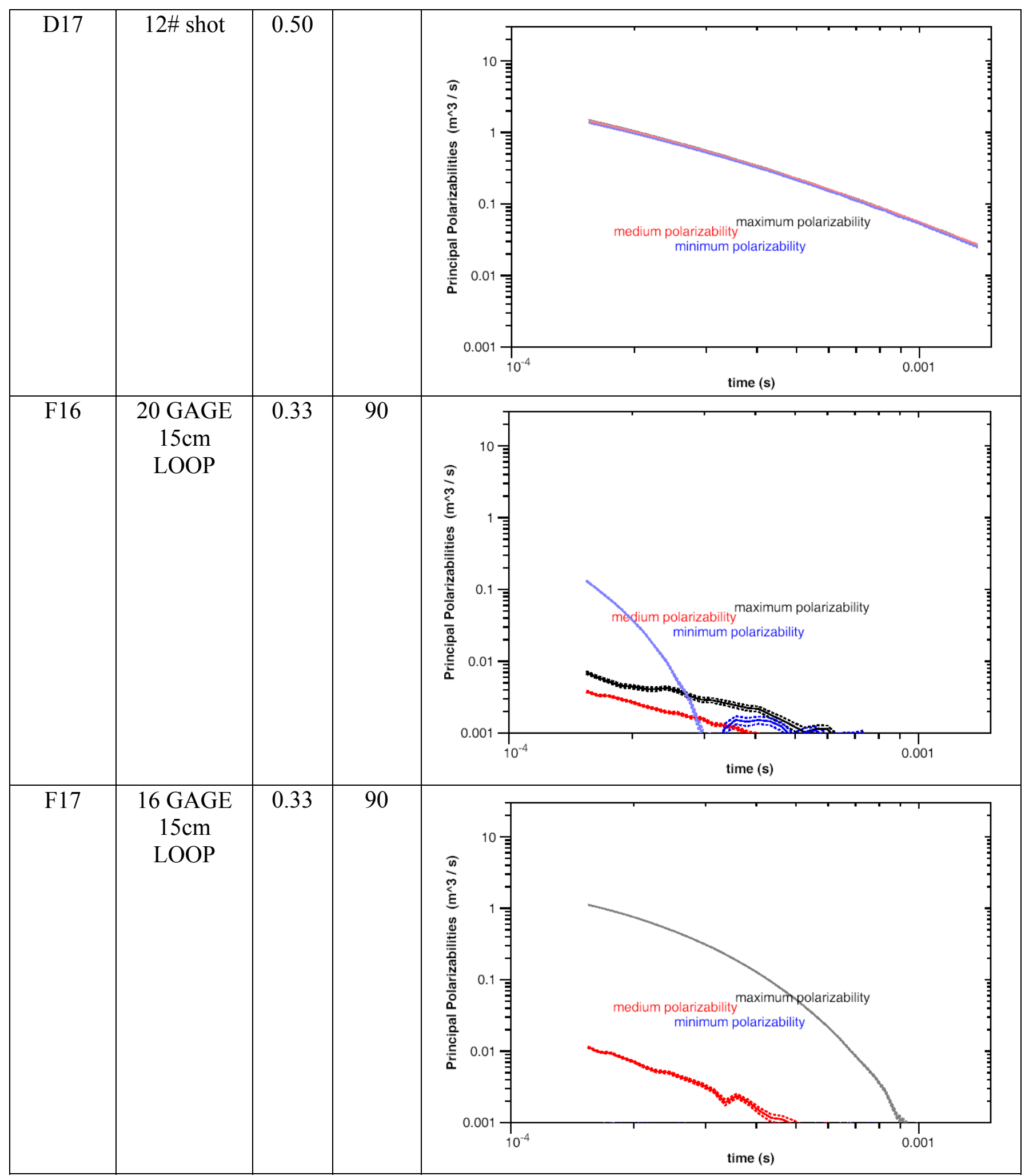




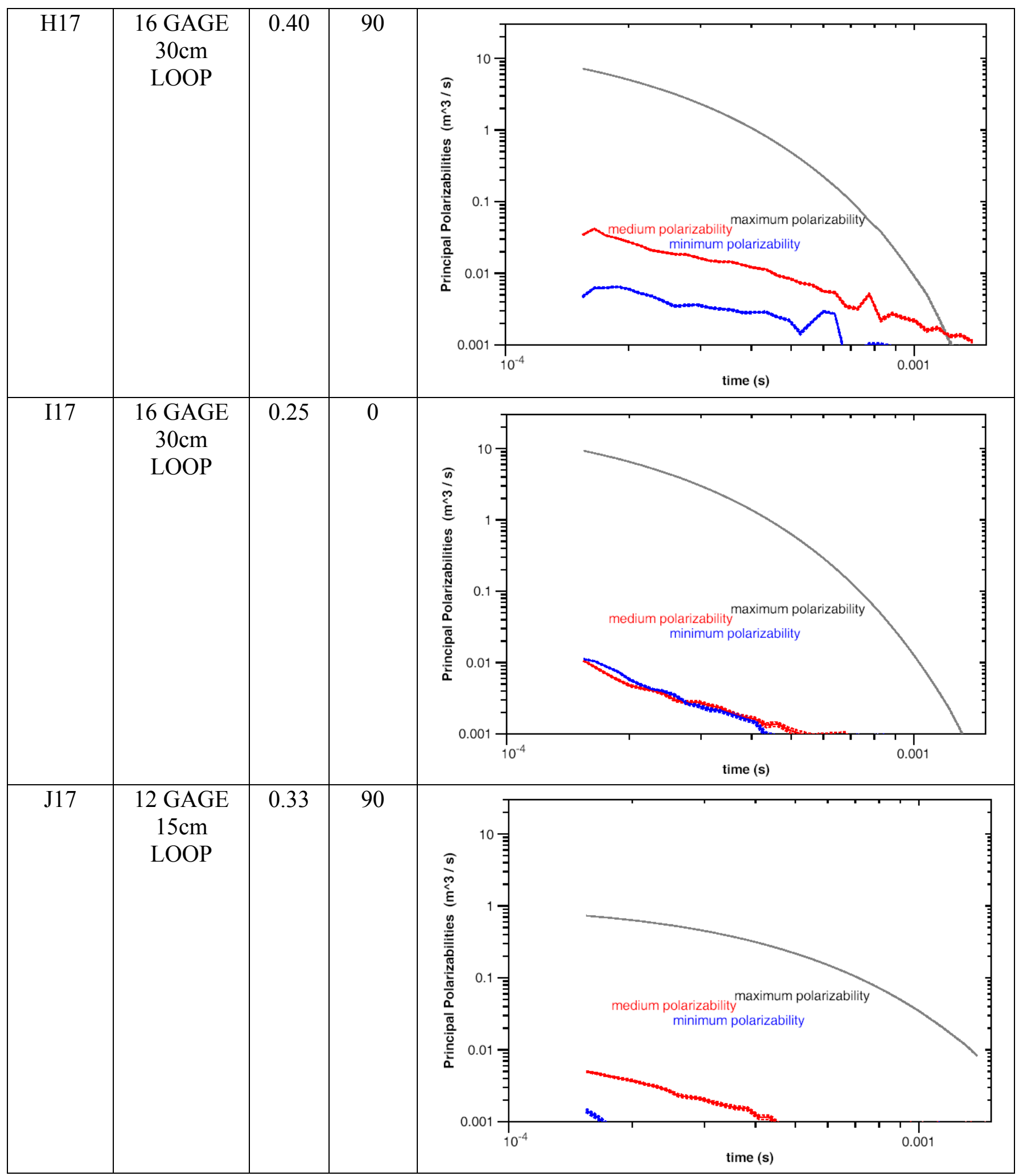




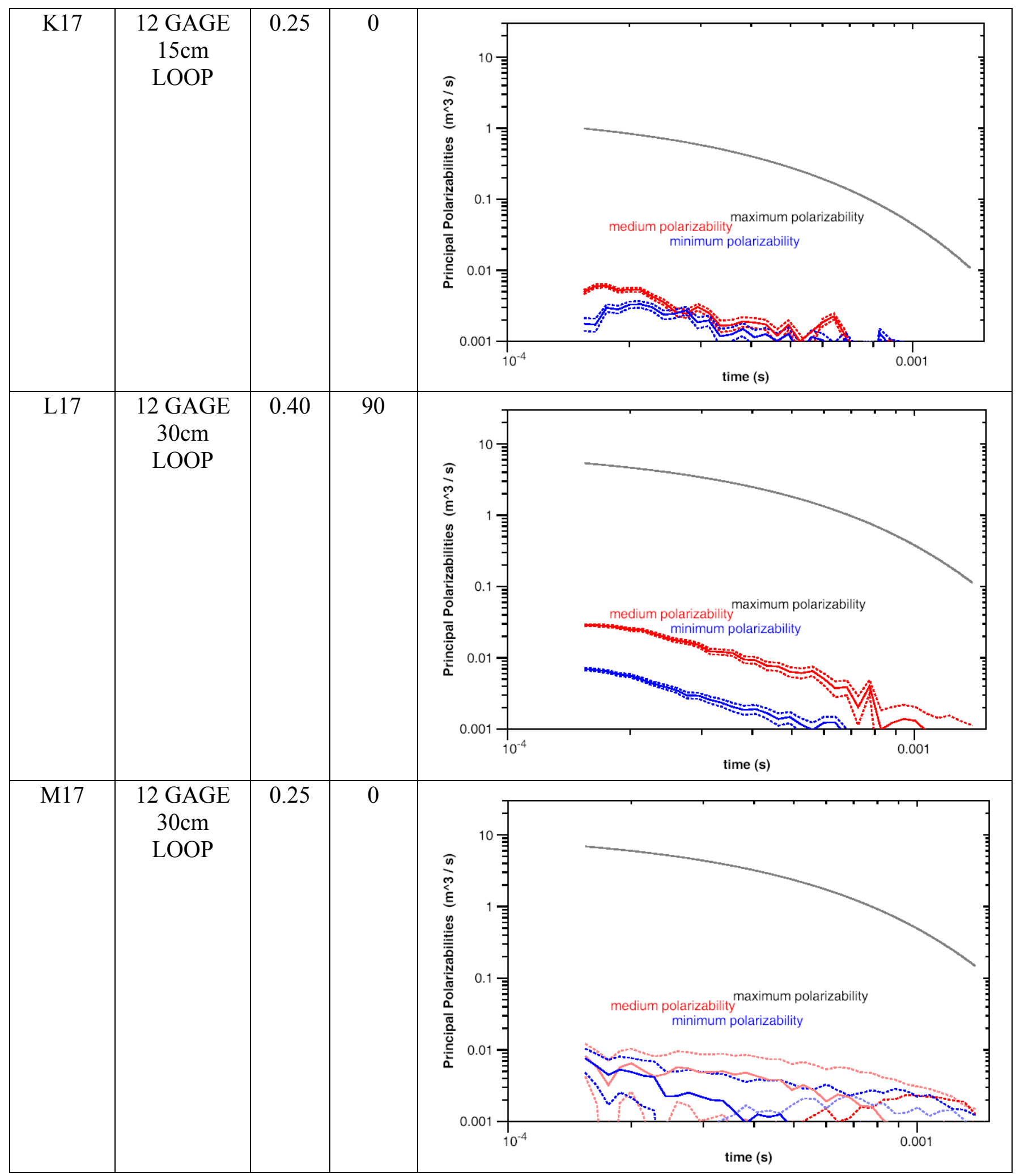


Table 2: Yuma Proving Ground - Blind Test Grid

\begin{tabular}{|c|c|}
\hline Probability & Cell Identification \\
\hline 1 & btg_a_01 \\
1 & btg_a_03 \\
1 & btg_a_04p \\
1 & btg_a_06 \\
1 & btg_a_08 \\
1 & btg_a_12m \\
1 & btg_a_14 \\
1 & btg_a_15 \\
1 & btg_a_17 \\
1 & btg_a_19 \\
\hline 1 & btg_b_01 \\
1 & btg_b_02 \\
1 & btg_b_04p \\
1 & btg_b_06 \\
1 & btg_b_07p \\
1 & btg_b_09 \\
0.6131 & btg_b_12 \\
1 & btg_b_15 \\
0.6556 & btg_b_16 \\
1 & btg_b_20 \\
\hline 1 & btg_c_01 \\
1 & btg_c_03 \\
1 & btg_c_04 \\
1 & btg_c_09 \\
1 & btg_c_11 \\
1 & btg_c_12 \\
1 & btg_c13m \\
1 & btg_c_17 \\
1 & btg_c_18 \\
1 & btg_c_19 \\
1 & btg_c_20 \\
\hline 1 & btg_d_01p \\
1 & btg_d_03 \\
1 & btg_d_04 \\
1 & btg_d_07 \\
0.4961 & btg_d_09p \\
0.5591 & btg_d_10p \\
0.9879 & btg_d_11p \\
1 & btg_d_12 \\
1 & btg_d_14p \\
1 & btg_d_15 \\
1 & btg_d_16 \\
& \\
\hline & \\
\hline & \\
\hline
\end{tabular}




\begin{tabular}{|c|c|}
\hline $\begin{array}{l}1 \\
1\end{array}$ & $\begin{array}{l}\text { btg_d_18 } 18 \\
\text { btg_d_19 }\end{array}$ \\
\hline 0.5814 & btg_e_01 \\
\hline 0.5844 & btg_e_02 \\
\hline 1 & btg_e_03 \\
\hline 0.6306 & btg_e $-55 \mathrm{~m}$ \\
\hline 1 & btg_e_08 \\
\hline 1 & btg $\mathrm{e}-10$ \\
\hline 1 & btg_e_11 \\
\hline 1 & btg $\mathrm{e}-12$ \\
\hline 1 & btg_e_13 \\
\hline 0.9761 & btg $\mathrm{e}-14$ \\
\hline 1 & btg_e_15 \\
\hline 1 & btg_e_16 \\
\hline 1 & btg_e 17 \\
\hline 1 & btg_e \\
\hline 1 & btg e $20 p$ \\
\hline 1 & btg_f_01 \\
\hline 1 & btg $\mathrm{f} 02 \mathrm{~h}$ \\
\hline 1 & btg_f_04 \\
\hline 1 & btg f 05 \\
\hline 1 & btg_f_08 \\
\hline 1 & btg_f_10 \\
\hline 1 & btg $\mathrm{f} 11 \mathrm{p}$ \\
\hline 0.9052 & btg_f_14 \\
\hline 1 & btg f 15 \\
\hline 0.9999 & btg_f_18h \\
\hline 1 & btg $\mathrm{f} 20$ \\
\hline 0.531 & btg_g_02 \\
\hline 1 & btg_g_03 \\
\hline 1 & btg_g_05 \\
\hline 1 & btg_g_10 \\
\hline 1 & btg_g_11 \\
\hline 1 & btg_g_12 \\
\hline 1 & btg_g_15 \\
\hline 1 & btg_g_16 \\
\hline 1 & btg_g_17 \\
\hline 1 & btg_g_18 \\
\hline 1 & btg_h_01 \\
\hline 0.9999 & btg_h $\_03 \mathrm{~m}$ \\
\hline 1 & btg_h_ 04 \\
\hline 1 & btg_h_05 \\
\hline 1 & btg_h_12 \\
\hline 1 & btg_h_13 \\
\hline 1 & btg_h_14 \\
\hline 1 & btg_h_20 \\
\hline
\end{tabular}




\begin{tabular}{|c|c|}
\hline 1 & btg_i 01 \\
\hline 1 & btg_i_02 \\
\hline 1 & btg_i_04 \\
\hline 1 & btg $\mathrm{i} 05$ \\
\hline 1 & btg $\mathrm{i} 07$ \\
\hline 1 & btg_i_10 \\
\hline 1 & btg_i $11 \mathrm{~m}$ \\
\hline 1 & btg_i ${ }_{i} 12$ \\
\hline 1 & btg_i_14 \\
\hline 1 & btg_i_15 \\
\hline 1 & btg_i_17 \\
\hline 1 & btg_i_18 \\
\hline 1 & btg_i_20 \\
\hline 1 & btg_j_05 \\
\hline 1 & btg_j_07 \\
\hline 1 & btg_j_09 \\
\hline 1 & btg $\vec{j} \_10 \mathrm{~m}$ \\
\hline 0.9364 & $b \operatorname{btg} j_{-} 11 \mathrm{~m}$ \\
\hline 1 & $\mathrm{btg} \overline{\mathrm{j}}_{-} 12$ \\
\hline 0.7599 & btg_j_13 \\
\hline 1 & btg $\mathrm{j}_{-} 15$ \\
\hline 1 & btg_j_17 \\
\hline 1 & btg_j_19 \\
\hline 1 & btg_j_20 \\
\hline 1 & btg_k_01 \\
\hline 1 & btg_k_03 \\
\hline 1 & btg_k_04 \\
\hline 1 & btg_k_-05 \\
\hline 0.5425 & btg_k_07 \\
\hline 0.9904 & btg $\mathrm{k} 11$ \\
\hline 1 & btg_k_12 \\
\hline 1 & btg_k_16 \\
\hline 1 & btg_k_17 \\
\hline 1 & btg_k_19 \\
\hline 0.505 & btg $102 p$ \\
\hline 1 & btg_1_04p \\
\hline 1 & btg_1_06 \\
\hline 1 & btg_1_07 \\
\hline 1 & btg_1_08 \\
\hline 1 & btg_1_09 \\
\hline 1 & btg_1_11 \\
\hline 1 & btg_1_12p \\
\hline 0.9807 & btg_1_16 \\
\hline 0.5871 & btg_1_17 \\
\hline 0.7633 & btg_1_19 \\
\hline 0.8082 & btg $1 \_20$ \\
\hline
\end{tabular}




\begin{tabular}{|c|c|}
\hline 1 & btg_m_01 \\
1 & btg_m_02 \\
0.7622 & btg_m_06 \\
1 & btg_m_07 \\
1 & btg_m_09 \\
1 & btg_m_11 \\
1 & btg_m_12 \\
1 & btg_m_13 \\
1 & btg_m_14 \\
0.877 & btg_m_16 \\
1 & btg_m_17 \\
1 & btg_m_18 \\
0.7829 & btg_m_20 \\
\hline 1 & btg_n_01 \\
0.7261 & btg_n_03 \\
1 & btg_n_05 \\
1 & btg_n_06 \\
1 & btg_n_09 \\
1 & btg_n_13 \\
1 & btg_n_14 \\
1 & btg_n_16 \\
0.9996 & btg_n_17 \\
1 & btg_n_19 \\
\hline 1 & btg_o_01 \\
1 & btg_o_02 \\
1 & btg_o_04 \\
1 & btg_o_05 \\
1 & btg_o_06 \\
1 & btg_o_08p \\
1 & btg_o_10p \\
1 & btg_o_11p \\
1 & btg_o_14 \\
0.6334 & btg_o_15 \\
1 & btg_o_18 \\
\hline 1 & btg_p_01 \\
0.6934 & btg_p_02m \\
0.6001 & btg__03p \\
1 & btg_p_04 \\
1 & btg_p_08 \\
1 & btg_p_09 \\
1 & btg_p_11 \\
1 & btg_p_12 \\
1 & btg_p_13 \\
1 & btg_p_15 \\
1 & btg_p_16 \\
1 & btg_p_17 \\
\hline & \\
\hline & \\
\hline & \\
\hline
\end{tabular}




\begin{tabular}{|c|c|}
\hline 1 & btg_p_20 \\
\hline 1 & btg_q_02 \\
1 & btg_q_03 \\
0.8878 & btg_q_04 \\
0.5236 & btg_q_05 \\
0.7798 & btg_q060 \\
0.5219 & btg_q_07p \\
1 & btg_q_09 \\
1 & btg_q_10 \\
1 & btg_q_11 \\
0.5923 & btg_q_13m \\
1 & btg_q_14 \\
1 & btg_q_15 \\
1 & btg_q_17 \\
1 & btg_q_19 \\
1 & btg_q_20 \\
\hline 1 & btg_r_01 \\
0.9849 & btg_r_02 \\
1 & btg_r_03 \\
0.4781 & btg__05m \\
0.3288 & btg_r_06 \\
1 & btg_r_07 \\
1 & btg_r_08 \\
0.997 & btg_r_09 \\
1 & btg_r_10 \\
1 & btg_r_11 \\
0.3466 & btg_r_14 \\
0.4386 & btg_r_16 \\
1 & btg_r_18 \\
0.3896 & btg_r_20 \\
\hline 1 & btg_s_04 \\
1 & btg_s_05 \\
1 & btg_s_13 \\
1 & btg_s_16 \\
1 & btg_s_17 \\
1 & btg_s_20 \\
\hline 1 & btg_t_01 \\
1 & btg_t_03 \\
0.7831 & btg_t_04 \\
0.8698 & btg_t_06 \\
1 & btg_t_07 \\
1 & btg_t_08 \\
1 & btg_t_10 \\
0.5821 & btg_t_11 \\
1 & btg_t_12 \\
1 & btg_t_14 \\
\hline & \\
\hline & \\
\hline & \\
\hline
\end{tabular}




\begin{tabular}{|l|l|}
\hline 1 & btg_t_16 \\
1 & btg_t_17 \\
\hline
\end{tabular}

